ESAIM: M2AN 48 (2014) 665-696

DOI: $10.1051 / \mathrm{m} 2 \mathrm{an} / 2013106$
ESAIM: Mathematical Modelling and Numerical Analysis

www.esaim-m2an.org

\title{
A POSITIVITY PRESERVING CENTRAL SCHEME FOR SHALLOW WATER FLOWS IN CHANNELS WITH WET-DRY STATES *
}

\author{
Jorge Balbás ${ }^{1}$ and Gerardo Hernandez-Duenas ${ }^{2}$
}

\begin{abstract}
We present a high-resolution, non-oscillatory semi-discrete central scheme for onedimensional shallow-water flows along channels with non uniform cross sections of arbitrary shape and bottom topography. The proposed scheme extends existing central semi-discrete schemes for hyperbolic conservation laws and enjoys two properties crucial for the accurate simulation of shallow-water flows: it preserves the positivity of the water height, and it is well balanced, i.e., the source terms arising from the geometry of the channel are discretized so as to balance the non-linear hyperbolic flux gradients. In addition to these, a modification in the numerical flux and the estimate of the speed of propagation, the scheme incorporates the ability to detect and resolve partially wet regions, i.e., wet-dry states. Along with a detailed description of the scheme and proofs of its properties, we present several numerical experiments that demonstrate the robustness of the numerical algorithm.
\end{abstract}

Mathematics Subject Classification. 76M12, 35L65.

Received June 7, 2012.

Published online January 20, 2014.

\section{INTRODUCTION}

Many geophysical and atmospheric flows are characterized by their large length scale relative to their depth (i.e., they are shallow). They are often modeled by the shallow water equations, a nonlinear hyperbolic conservation law with geometric source terms that results from the cross sectional averaging of Euler equations. Non-trivial (nearly) steady flows are of particular interest as they arise commonly in nature, e.g., channel and strait flows, flows through mountain passes, etc. The accurate approximation and simulation of these require the delicate balance between the nonlinear flux gradients and the geometric source terms of the hyperbolic system.

Recent years have seen a rapidly growing interest in the development of numerical methods for shallow water systems in various numerical frameworks [4,5,15,18,20,21,24-29], see also the recent book [8] and references therein. Works involving shallow-water flows in variable geometry include $[14,19,31]$ where an upwind scheme for the single layer shallow water is derived and generalized to rectangular channel flows, [17] where the model

\footnotetext{
Keywords and phrases. Hyperbolic systems of conservation and balance laws, semi-discrete schemes, Saint-Venant system of Shallow Water equations, non-oscillatory reconstructions, channels with irregular geometry.

* Work supported in part by NSF award number DMS 0609766, and by Conacyt \#16014\%.

1 Department of Mathematics. California State University, Northridge. 18111 Nordhoff St. Northridge, CA 91330-8313, USA. jorge.balbas@csun.edu

2 Department of Mathematics, University of Wisconsin - Madison. 480 Lincoln Dr., Madison, Wi 53706-1325, USA.

hernande@math.wisc.edu
} 

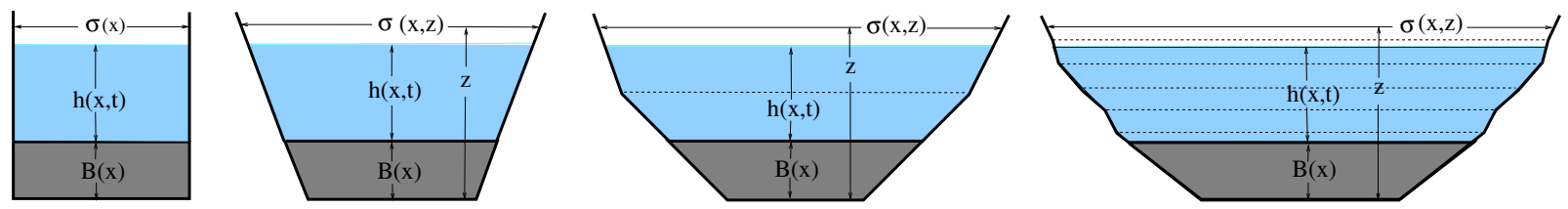

FigURE 1. Schematic of channel cross section.

was generalized to arbitrary cross-sectional areas, and [10] where the Q-scheme introduced in [9] is used to solve the two layer shallow water system, and the central schemes introduced in [12,32]. Well-balanced and positivity preserving central and central-upwind schemes have been derived in [5,21].

In this paper we present a high-resolution semi-discrete central scheme for shallow-water flows along channels with non-uniform cross-sections of arbitrary shape and bottom topography. The scheme is based on the higher order version of Rusanov's numerical flux introduced in [23], and it generalizes the central schemes in [5, 21] to the general variable cross section shallow water equations treated in [17]. The interplay between the bottom topography and the varying width of the channel affects and controls the flow. Numerical schemes for shallowwater flows must, therefore, pay special attention to the discretization of the channel's geometry so as to make it consistent with the well-balance and positivity properties sought. In this case, we ensure the well-balance property by describing the channel's floor and walls with piecewise linear segments, a choice that renders piecewise trapezoidal cross sections (see Fig. 1).

The paper is structured as follows, in Section 2 we provide a description of the system (2.1), its properties, and the challenges that these properties pose for computing numerical solution. In Section 3 we describe the proposed numerical scheme and prove that it preserves the positivity of the water height, it is well-balanced, i.e., it recognizes and preserves the steady-state of rest, and that it is capable of identifying and resolving wet-dry regions. Numerical solutions for a variety of flow regimes are presented in Section 4, validating the scheme's accuracy and robustness and demonstrating its ability to simulate a wide range of flows.

\section{THE MODEL}

The shallow water equations for flows through channels with variable cross-section are given by, [14,17],

$$
\begin{aligned}
& \frac{\partial A}{\partial t}+\frac{\partial Q}{\partial x}=0 \\
& \frac{\partial Q}{\partial t}+\frac{\partial}{\partial x}\left(\frac{Q^{2}}{A}+I_{1}\right)=I_{2}-g \sigma_{B} h B^{\prime}
\end{aligned}
$$

where $h$ denotes the depth of the layer, $u$ the the cross-sectional velocity, $B(x)$ the bottom topography, $\sigma(x, z)$ the width of the channel, $A=\int_{B}^{B+h} \sigma(x, z) \mathrm{d} z$ is the cross-sectional wet area, $Q=A u$ is the flow rate or discharge, $\sigma_{B}(x)=\sigma(x, B(x))$ denotes the channel width at $z=B(x)$, and $g$ the acceleration of gravity. The terms $I_{1}(x)$ and $I_{2}(x)$ that appear in the flux and the source term stand, respectively, for the integrals

$$
I_{1}(x)=g \int_{B}^{w}(w-z) \sigma(x, z) \mathrm{d} z=A p, \quad \text { and } I_{2}(x)=g \int_{B}^{w}(w-z) \sigma_{x}(x, z) \mathrm{d} z
$$

where $w$ denotes the total water elevation, $w=h+B$, and $p$ the cross-sectional average of the hydrostatic pressure, (See Fig. 1). 


\subsection{Properties of the system}

In quasilinear form the system reads

$$
\left(\begin{array}{l}
A \\
Q
\end{array}\right)_{t}+\left(\begin{array}{cc}
0 & 1 \\
c^{2}-u^{2} & 2 u
\end{array}\right)\left(\begin{array}{l}
A \\
Q
\end{array}\right)_{x}=\left(\begin{array}{c}
0 \\
c^{2}\left(h I_{3}-\sigma_{B} B^{\prime}\right)
\end{array}\right)
$$

where $I_{3}(x)=\frac{1}{h} \int_{B}^{w} \sigma_{x}(x, z) \mathrm{d} z$ is the averaged width variation, and $c^{2}=g A / \sigma_{T}$, where $\sigma_{T}=\sigma(x, h+B)$ is the width of the channel at the water surface. Notice that $c^{2}$ reduces to the familiar expression $c^{2}=g h$ for rectangular channels.

The system (2.1) is hyperbolic, with eigenvectors and eigenvalues

$$
R=\left(\begin{array}{cc}
1 & 1 \\
u-c & u+c
\end{array}\right) \quad \Lambda=\left(\begin{array}{cc}
u-c & 0 \\
0 & u+c
\end{array}\right)
$$

and the flows it describes are characterized by the nondimensional Froude number $F$, where $F^{2}=\frac{u^{2}}{c^{2}}$. The Froude number captures the essential non-linearity of the flow; a flow is said to be subcritical for $F^{2}<1$ and supercritical for $F^{2}>1$, [13,17].

The system is endowed with an entropy function

$$
\mathcal{E}=A\left(\frac{1}{2} u^{2}+g(h+B)\right)-I_{1}
$$

which satisfies the inequality, [4,27]

$$
\frac{\partial \mathcal{E}}{\partial t}+\frac{\partial}{\partial x}\left[Q\left(\frac{1}{2} u^{2}+g(h+B)\right)\right] \leq 0
$$

See also $[5,17]$ for the entropy inequality for channels with cross-sectional area variation and arbitrary geometry respectively. Strict hyperbolicity is lost for $h=0$, when eigenvectors coincide, representing a so-called "dry state".

\subsection{Steady-State solutions}

Smooth steady-state solutions are characterized by two invariants, the flow rate $Q$, and $E$, the energy per unit of cross sectional area

$$
Q \equiv A u=\text { Const. }, \quad E \equiv \frac{1}{2} u^{2}+g(h+B)=\text { Const. }
$$

among which it is easy to recognize the steady state of rest

$$
u=0, \quad h+B=\text { Const. }
$$

Exact solutions - to machine precision - for smooth steady-states can be found by solving (using a rootfinding method),

$$
E=\frac{1}{2} \frac{Q^{2}}{A^{2}}+g(h+B),
$$

with prescribed boundary conditions $Q_{\text {in }}$ at the inflow boundary and $h+B=w_{\text {out }}$ at the outflow. And non smooth steady-states - characterized by a stationary jump - by prescribing, in addition to $Q_{\text {in }}$ and $w_{\text {out }}$, the depth of the flow at some point between the inflow boundary and the location of the discontinuity. A detailed discussion about these solutions for channels with (nonuniform) rectangular cross-sections is presented in $[2,3,13]$. 
In the case of a straight channel, smooth steady solutions satisfy (here $h^{\prime}=h_{x}$ )

$$
\left(F^{2}-1\right) h^{\prime}=B^{\prime} .
$$

That is, at the crest, i.e., $B^{\prime}=0$, the solution is either critical $\left(F^{2}=1\right)$ or symmetric $\left(h^{\prime}=0\right)$. If the channel is rectangular with variable cross sectional width $\sigma=\sigma(x)$, then

$$
\left(F^{2}-1\right) h^{\prime}=B^{\prime}-\frac{h \sigma^{\prime}}{\sigma} F^{2}
$$

Implying that if the crest $\left(B^{\prime}=0\right)$ and the throat $\left(\sigma^{\prime}=0\right)$ occur at the same point, the right hand side of $(2.9)$ vanishes there and the flow is either symmetric or reaches criticality at that point. Otherwise, criticality occurs where

$$
B^{\prime}=\frac{h}{\sigma} F^{2} \sigma^{\prime}
$$

which occurs somewhere between the crest and the throat, [5]. For the channels with arbitrary geometry considered in this work, steady-state solutions satisfy, [17],

$$
\left(F^{2}-1\right) h^{\prime}=\left(1-\frac{\left(\sigma_{T}-\sigma_{B}\right) F^{2}}{\sigma_{T}}\right) B^{\prime}-\frac{h I_{3}}{\sigma_{T}} F^{2},
$$

and their exact solution can be calculated (to machine precession) with a root finding method by imposing the appropriate boundary conditions, $Q_{\text {in }}$ and $w_{\text {out }}$ for smooth flows, and those plus $h$ (or $w$ ) at some other point for non smooth flows.

\subsection{Numerical simulation of shallow-water flows}

The nonlinear flux in (2.1) together with the properties described above and the interplay between the flow quantities and the channel geometry pose a number of challenges when computing the numerical solution of (2.1): The nonlinearity of the flux implies that the balance law admits discontinuous solutions, and requires robust numerical schemes that are suitable for calculating discontinuous flows. Another difficulty arises when computing solutions where $h \rightarrow 0$ (e.g., dam break), round off errors may cause the depth of the water layer, $h$, to become negative leading to the loss of hyperbolicity and causing the computation to fail. Positivity preserving schemes have the desirable property that if the data has positive (non-negative) depth, so does the numerical solution, and they enjoy enhanced stability near dry states. Changes in the solution of (2.1) in time arise when flux gradients are out of balance with the source terms, so numerical schemes that are able to recognize and respect such a balance often give superior results when computing near steady-state flows. Perfectly recognizing such a balance may not always be possible, and schemes that respect steady-state solutions either exactly or to the order of the numerical approximation are often called "well-balanced". However, the ability of a scheme to detect steady-state solutions and to converge to them, and to preserve the positivity of the water layer may not be sufficient to preserve steady-states of rest exactly in all scenarios. For instance, in a channel with piecewise discontinuous bottom topography one could encounter two states of rest with the deeper part of the channel holding a positive water layer (wet) and the higher ground being dry. The evolution of such wet-dry front could create spurious oscillations at the interface between the two states leading to instabilities. In Section 3 we take all these challenges into consideration and we incorporate into our central scheme the necessary techniques to properly address them.

\section{NUMERICAL SCHEME}

In this section we construct a central scheme for the accurate simulation of shallow-water flows described by the balance law (2.1). In particular, we seek a scheme that is positivity preserving and well-balanced. The scheme extends previous works in $[5,20-22]$ to flows along channels with variable geometry. This extension is 
not trivial; the varying geometry of the channel leads to fluxes and source terms that require the approximation of integral terms, making the balance of them more difficult; while in channels with constant width $(\sigma \equiv 1)$, well-balancing may be accomplished solely by choosing an appropriate discretization of the source term, in the variable geometry case, the conserved variables $A=\int_{B}^{B+h} \sigma(x, z) \mathrm{d} z$ and $Q=A u$ depend on the geometry $\sigma$, which renders steady-state preservation and positivity more strongly coupled with, for example, the polynomial reconstruction of the conserved variables.

To this end, it is convenient - following [22] - to reformulate (2.1) in terms of the total elevation of the free water layer, $w=h+B$ and its total area, $A^{T}=A+\int_{0}^{B} \sigma(x, z) \mathrm{d} z$, that is

$$
\begin{aligned}
& \frac{\partial A^{T}}{\partial t}+\frac{\partial Q}{\partial x}=0 \\
& \frac{\partial Q}{\partial t}+\frac{\partial}{\partial x}\left(\frac{Q^{2}}{A^{T}-\int_{0}^{B} \sigma(x, z) \mathrm{d} z}+I_{1}\right)=I_{2}-g \sigma_{B}(w-B) B^{\prime}
\end{aligned}
$$

This formulation allows the numerical scheme to detect changes (or the lack of them) in the total water elevation, $w$, which in turn, facilitates ensuring preservation of steady-states of rest.

\subsection{Semi-discrete central formulation}

We write the modified balance law, (3.1), in the more general form

$$
v_{t}+f(v)_{x}=S(v, x)
$$

with

$$
v=\left(\begin{array}{c}
A^{T} \\
Q
\end{array}\right), \quad f(v)=\left(\frac{Q^{2}}{\frac{Q}{A^{T}-\int_{0}^{B} \sigma(x, z) \mathrm{d} z}+I_{1}}\right),
$$

on the left hand side, and with the source term

$$
S=\left(\begin{array}{c}
0 \\
I_{2}-g \sigma_{B}(w-B) B^{\prime}
\end{array}\right) .
$$

Fixing a spatial scale $\Delta x$, we partition the solution domain into the grid cells $I_{j}:=\left[x_{j}-\frac{\Delta x}{2}, x_{j}+\frac{\Delta x}{2}\right]$, and denote by $\bar{v}_{j}(t)$ the cell average of $v(x, t)$ over the cell $I_{j}$,

$$
\bar{v}_{j}(t)=\frac{1}{\Delta x} \int_{x_{j-\frac{1}{2}}}^{x_{j+\frac{1}{2}}} v(x, t) \mathrm{d} x .
$$

Integrating equation (3.2) over the cells $\left\{I_{j}\right\}_{j}$, we obtain the semidiscrete formulation

$$
\frac{\mathrm{d}}{\mathrm{d} t} \bar{v}_{j}(t)+\frac{1}{\Delta x}\left(f\left(v\left(x_{j+\frac{1}{2}}, t\right)\right)-f\left(v\left(x_{j-\frac{1}{2}}, t\right)\right)\right)=\frac{1}{\Delta x} \int_{x_{j-\frac{1}{2}}}^{x_{j+\frac{1}{2}}} S(v(x, t), x) \mathrm{d} x,
$$

which is approximated by

$$
\frac{\mathrm{d}}{\mathrm{d} t} \bar{v}_{j}(t)=-\frac{H_{j+\frac{1}{2}}-H_{j-\frac{1}{2}}}{\Delta x}+\frac{1}{\Delta x} \int_{x_{j-\frac{1}{2}}}^{x_{j+\frac{1}{2}}} S(v, x) \mathrm{d} x
$$

where the flux at the cell interfaces, $f\left(v\left(x_{j \pm \frac{1}{2}}\right), t\right)$, is approximated by the numerical flux $H_{j \pm \frac{1}{2}}(t)$ given by, [23],

$$
H_{j \pm \frac{1}{2}}(t)=\frac{f\left(v_{j \pm \frac{1}{2}}^{+}(t)\right)+f\left(v_{j \pm \frac{1}{2}}^{-}(t)\right)}{2}-\frac{a_{j \pm \frac{1}{2}}}{2}\left(v_{j \pm \frac{1}{2}}^{+}(t)-v_{j \pm \frac{1}{2}}^{-}(t)\right) .
$$


Here, the interface point-values $v_{j \pm \frac{1}{2}}^{ \pm}(t)$ are recovered from the cell averages via a non-oscillatory piecewise polynomial reconstruction

$$
v_{j+\frac{1}{2}}^{-}:=p_{j}\left(x_{j+\frac{1}{2}}\right), \quad \text { and } \quad v_{j+\frac{1}{2}}^{+}:=p_{j+1}\left(x_{j+\frac{1}{2}}\right),
$$

and $a_{j \pm \frac{1}{2}}$ stands for an estimate for the maximum wave speed of the balance law, approximated by

$$
a_{j \pm \frac{1}{2}}=\max \left\{\left|u_{j \pm \frac{1}{2}}^{-}\right|+c_{j \pm \frac{1}{2}}^{-},\left|u_{j \pm \frac{1}{2}}^{+}\right|+c_{j \pm \frac{1}{2}}^{+}\right\} .
$$

In addition to the non-oscillatory polynomial reconstruction - which must ensure the positivity of the free surface - and the wave speed estimate, the implementation of the scheme requires a discretization of the source term integral on the right hand side of (3.7) that balances the numerical fluxes, a mechanism to identify and resolve wet-dry regions, and an evolution routine to update the resulting ODE system. The description of all these ingredients follows.

\subsection{Positivity preserving non-oscillatory reconstruction}

In order to recover the interface point values $v_{j \pm \frac{1}{2}}(t)$ from the cell averages $\bar{v}_{j}(t)$, we seek a piecewise polynomial reconstruction

$$
v(x, t)=R(x ; \bar{v}(t)):=\sum_{j} p_{j}(x) .
$$

This reconstruction procedure is at the heart of high-resolution non-oscillatory central schemes, and requires the coefficients of the polynomials $\left\{p_{j}(x)\right\}$ to be determined so that $R(x ; \bar{v}(t))$ satisfies the following essential properties:

- $\mathcal{P}_{1}$ - Conservation of cell averages: $\bar{p}_{j}(x)=\bar{v}_{j}(t)$.

- $\mathcal{P}_{2}-$ Accuracy: $R(x ; \bar{v}(t))=v(x, t)+\mathcal{O}\left((\Delta x)^{2}\right)$ (in smooth regions).

- $\mathcal{P}_{3}$ - Non-oscillatory behavior of $\sum_{j} p_{j}(x)$.

- $\mathcal{P}_{4}$ - Flux gradient and source balancing: the interface values of the total area $A^{T}$ (and those of $w$ ) must be reconstructed so as to satisfy

$$
w_{j+\frac{1}{2}}^{ \pm}=\text {Const. }
$$

when the data is that of a steady-state of rest $(2.6), \bar{w}_{j}=$ Const..

- $\mathcal{P}_{5}$ - Positivity: the reconstructed values $w_{j \pm \frac{1}{2}}^{ \pm}(t)$ must yield $h_{j \pm \frac{1}{2}}^{ \pm}(t) \geq 0$, so as to ensure the positivity of $\bar{h}_{j}(t+\Delta t)$.

To this end, we choose

$$
p_{j}(x)=\bar{v}_{j}+v_{j}^{\prime}\left(x-x_{j}\right),
$$

with the limited slopes $v_{j}^{\prime}$ calculated as, [30],

$$
v_{j}^{\prime}=\frac{1}{\Delta x} \operatorname{minmod}\left(\alpha \Delta_{-} \bar{v}_{j}, \Delta_{0} \bar{v}_{j}, \alpha \Delta_{+} \bar{v}_{j}\right),
$$

where $1 \leq \alpha<2$, and

$$
\operatorname{minmod}\left(x_{1}, x_{2}, x_{3}, \ldots, x_{k}\right)=\left\{\begin{array}{lll}
\min _{j}\left(x_{j}\right) & \text { if } & x_{j}>0 \forall j \\
\max _{j}\left(x_{j}\right) & \text { if } & x_{j}<0 \forall j \\
0 & \text { otherwise }
\end{array}\right.
$$




\subsubsection{Steady-state of rest and positivity - properties $\mathcal{P}_{4}$ and $\mathcal{P}_{5}$}

This minmod reconstruction procedure will satisfy properties $\mathcal{P}_{1}-\mathcal{P}_{3}$ above when applied to any (smooth or non-smooth) piecewise data - property $\mathcal{P}_{3}$ is characterized by the TVD property for scalar hyperbolic conservation laws, [30]. However, in order to enforce $\mathcal{P}_{4}$ and $\mathcal{P}_{5}$, the reconstruction is applied to $Q$ and $w-$ whose cell averages are recovered from those of $A^{T}$ by deconvolving $\bar{A}_{j}^{T}=\int_{0}^{\bar{w}_{j}} \sigma\left(x_{j}, z\right) \mathrm{d} z$, which reduces to solving a quadratic equation when $\sigma\left(x_{j}, z\right)$ is replaced by the piecewise linear approximation illustrated in Figure 1. We refer the reader to Appendix B for the details of this deconvolution.

Reconstructing the pointvalues from the data $\left\{\bar{w}_{j}\right\}$ instead of $\left\{\bar{A}_{j}^{T}\right\}$ the well-balance property, $\mathcal{P}_{4}$, is trivially satisfied - as the minmod reconstruction will yield zero derivatives on the constant data $\left\{\bar{w}_{j}\right\}$, but not the positivity of $h_{j \pm \frac{1}{2}}^{ \pm}$. As illustrated in Figure 2, the different signs of the finite differences compared by the minmod limiter may lead to zero numerical derivatives and render interface values $w_{j \pm \frac{1}{2}}^{ \pm}$smaller than the corresponding values of the bottom topography $B_{j \pm \frac{1}{2}}$. To prevent this numerical artifact, we follow [21] and check the minmod reconstructed pointvalues $w_{j \pm \frac{1}{2}}^{ \pm}$, correcting them - if necessary - as follows

$$
\text { if } w_{j-\frac{1}{2}}^{+}<B_{j-\frac{1}{2}} \quad \Longrightarrow \quad w_{j}^{\prime}=2\left(\bar{w}_{j}-B_{j-\frac{1}{2}}\right) \quad \Longrightarrow \quad\left\{\begin{array}{l}
w_{j-\frac{1}{2}}^{+}=B_{j-\frac{1}{2}} \\
w_{j+\frac{1}{2}}^{-}=\bar{w}_{j}+\frac{1}{2} w_{j}^{\prime}
\end{array}\right.
$$

else

$$
\text { if } w_{j+\frac{1}{2}}^{-}<B_{j+\frac{1}{2}} \quad \Longrightarrow \quad w_{j}^{\prime}=2\left(B_{j+\frac{1}{2}}-\bar{w}_{j}\right) \quad \Longrightarrow \quad\left\{\begin{array}{l}
w_{j+\frac{1}{2}}^{-}=B_{j+\frac{1}{2}} \\
w_{j-\frac{1}{2}}^{+}=\bar{w}_{j}-\frac{1}{2} w_{j}^{\prime},
\end{array}\right.
$$

which yields

$$
h_{j+\frac{1}{2}}^{-}:=w_{j+\frac{1}{2}}^{-}-B_{j+\frac{1}{2}} \geq 0
$$

and

$$
h_{j-\frac{1}{2}}^{+}:=w_{j-\frac{1}{2}}^{+}-B_{j-\frac{1}{2}} \geq 0 .
$$

The pointvalues of the total area, $A_{j \pm \frac{1}{2}}^{T, \pm}$, are then recovered from these by integrating the interface width,

$$
A_{j \pm \frac{1}{2}}^{T, \pm}=\int_{0}^{w_{j \pm \frac{1}{2}}^{ \pm}} \sigma\left(x_{j \pm \frac{1}{2}}, z\right) \mathrm{d} z
$$

\subsubsection{Regularization of flow velocity and discharge for small $A$}

We shall remark however that while this modified minmod reconstruction will ensure the positivity of the water height at the cell interfaces, these pointvalues may still be very small (i.e., arbitrarily close to zero) and may lead to large values of the velocity of the flow, $u$, or, equivalently, of the term $\frac{Q^{2}}{A^{T}-\int_{0}^{B(x)} \sigma(x, z) \mathrm{d} z}=Q u$ in the second component of the flux $f$ in (3.3). In order to prevent this, after reconstructing $Q, w$, and $A^{T}$ at the cell interfaces, we use the regularization technique suggested by [21],

$$
u_{j \pm \frac{1}{2}}^{ \pm}=\frac{\sqrt{2} Q_{j \pm \frac{1}{2}}^{ \pm} A_{j \pm \frac{1}{2}}^{ \pm}}{\sqrt{\left(A_{j \pm \frac{1}{2}}^{ \pm}\right)^{4}+\max \left(\left(A_{j \pm \frac{1}{2}}^{ \pm}\right)^{4}, \delta^{4}\right)}}
$$

with

$$
A_{j \pm \frac{1}{2}}^{ \pm}=\int_{B_{j \pm \frac{1}{2}}}^{w_{j \pm \frac{1}{2}}^{ \pm}} \sigma_{j \pm \frac{1}{2}}(z) \mathrm{d} z, \quad \sigma_{j \pm 1 / 2}(z):=\sigma\left(x_{j \pm 1 / 2}, z\right)
$$




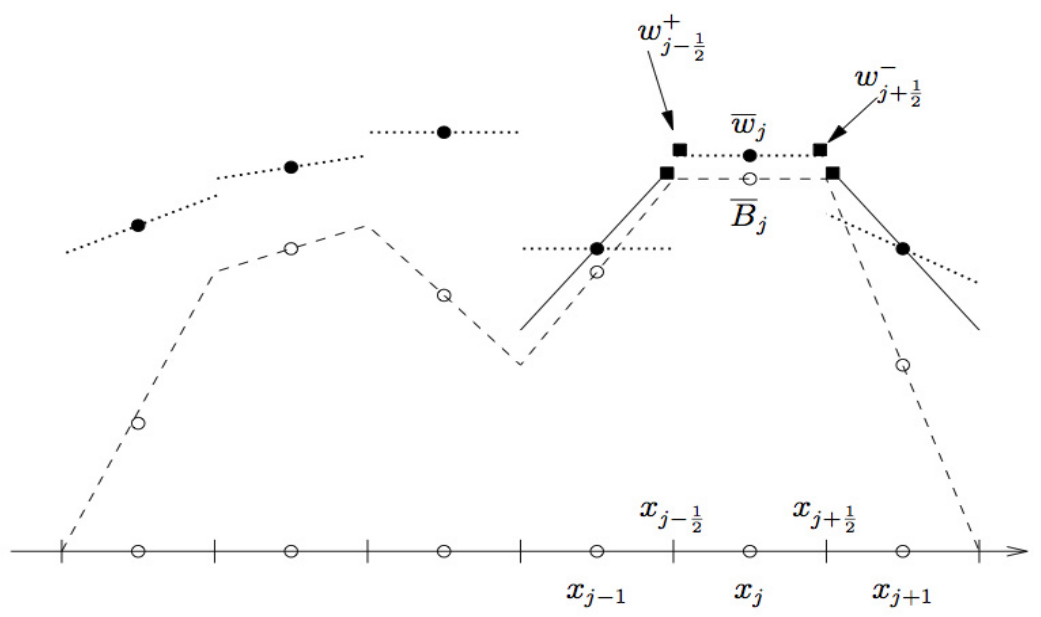

FiguRE 2. Modified reconstruction of total water height, $w$, over the piecewise linear approximation of bottom topography (dashed line). The minmod reconstruction is depicted by dotted lines over cell averages (black dots), the modified reconstruction is depicted by a black solid line, the interface pointvalues of $w, w_{j \pm \frac{1}{2}}$, are depicted by black squares.

And then recalculate the interface values of the discharge as

$$
Q_{j \pm \frac{1}{2}}^{ \pm}=A_{j \pm \frac{1}{2}}^{ \pm} u_{j \pm \frac{1}{2}}^{ \pm}
$$

The value of $\delta$ was empirically determined, usually choosing $\delta=5 \times 10^{-3}$ in this paper.

\subsection{Well balance}

If, at time $t$, the computed flow variables satisfy the steady-state conditions of a lake at rest (2.6), i.e., $\bar{w}_{j}=$ Const. and $\bar{Q}_{j}=0$ for all $j$, the reconstructed pointvalues $A_{j \pm \frac{1}{2}}^{T, \pm},(3.19)$, will trivially yield

$$
\frac{d \bar{A}_{j}^{T}(t)}{\mathrm{d} t}=0, \quad \forall j
$$

and thus $\bar{A}_{j}^{T}(t+\Delta t)=\bar{A}_{j}^{T}(t)$ as desired. This well-balance property allows the scheme to better handle near steady-state flows $[4,18,24,31]$. Satisfying also the well-balance property for $\bar{Q}_{j}(t)$ requires additional considerations; the cell average of the source term, $\bar{S}_{j}$, in (3.4) needs to be discretized so as to balance the numerical fluxes (3.8). In order to find such discretezation, we start by writing the second component of the numerical flux difference in (3.7) for the rest conditions (2.6). Under these conditions, noting that the minmod reconstruction of $Q$ will trivially yield $Q_{j \pm \frac{1}{2}}^{ \pm}=0$, this difference amounts to

$$
\begin{aligned}
\frac{H_{j+\frac{1}{2}}^{Q}-H_{j-\frac{1}{2}}^{Q}}{\Delta x} & =\frac{1}{2 \Delta x}\left[\left(f^{Q}\left(v_{j+\frac{1}{2}}^{+}\right)+f^{Q}\left(v_{j+\frac{1}{2}}^{-}\right)\right)-\left(f^{Q}\left(v_{j-\frac{1}{2}}^{+}\right)+f^{Q}\left(v_{j-\frac{1}{2}}^{-}\right)\right)\right] \\
& =\frac{g}{\Delta x}\left[\int_{B_{j+\frac{1}{2}}}^{w_{j+\frac{1}{2}}}\left(w_{j+\frac{1}{2}}-z\right) \sigma\left(x_{j+\frac{1}{2}}, z\right) \mathrm{d} z-\int_{B_{j-\frac{1}{2}}}^{w_{j-\frac{1}{2}}}\left(w_{j-\frac{1}{2}}-z\right) \sigma\left(x_{j-\frac{1}{2}}, z\right) \mathrm{d} z\right],
\end{aligned}
$$


and it should balance the cell average of the source term in (3.7), that is

$$
\bar{S}_{j}=\frac{g}{\Delta x} \int_{x_{j-\frac{1}{2}}^{+}}^{x_{j+\frac{1}{2}}^{-}}\left[\int_{B}^{w}(w-z) \sigma_{x}(x, z) \mathrm{d} z-\sigma_{B}(x)(w-B) B^{\prime}\right] \mathrm{d} x .
$$

Thus, the well balance property of the scheme amounts to devising a (high-order) discrete analog of the integral in (3.25) that cancels the discretized counterpart of (3.24) exactly when $w=$ Const., and $u=0$. To this end, we propose:

Proposition 3.1. Let $B_{j \pm \frac{1}{2}}=B\left(x_{j \pm \frac{1}{2}}\right)$, and $\sigma_{j \pm \frac{1}{2}}(z)=\sigma\left(x_{j \pm \frac{1}{2}}, z\right)$ be the topography and geometry at the interfaces $x_{j \pm \frac{1}{2}}$, and define the following approximation of the cell average $\bar{S}_{j}$ in (3.25)

$$
\frac{1}{\Delta x} \int_{x_{j-\frac{1}{2}}}^{x_{j+\frac{1}{2}}} \int_{B}^{w} g(w-z) \sigma_{x}(x, z) \mathrm{d} z \mathrm{~d} x \approx \frac{1}{\Delta x} \frac{g}{2}\left(\int_{B_{j-\frac{1}{2}}}^{w_{j-\frac{1}{2}}^{+}}+\int_{B_{j+\frac{1}{2}}}^{w_{j+\frac{1}{2}}^{-}}\right)\left(\bar{w}_{j}-z\right) \Delta \sigma_{j}(z) \mathrm{d} z,
$$

and

$$
\frac{1}{\Delta x} \int_{x_{j-\frac{1}{2}}}^{x_{j+\frac{1}{2}}} g \sigma_{B}(x) h B_{x} \mathrm{~d} x \approx \frac{g}{\Delta x} \int_{B_{j-\frac{1}{2}}}^{B_{j+\frac{1}{2}}}\left(\bar{w}_{j}-z\right) \bar{\sigma}_{j}(z) \mathrm{d} z,
$$

where $w_{j \mp \frac{1}{2}}^{ \pm}$are the reconstructed pointvalues of $w=h+B$ at the interfaces $x_{j \pm \frac{1}{2}}$ (in the interior of the cell $I_{j}$ ),

$$
\bar{w}_{j}=\frac{w_{j-\frac{1}{2}}+w_{j+\frac{1}{2}}}{2}, \quad \Delta \sigma_{j}(z)=\sigma_{j+\frac{1}{2}}(z)-\sigma_{j-\frac{1}{2}}(z), \quad \text { and } \quad \bar{\sigma}_{j}(z)=\frac{\sigma_{j-\frac{1}{2}}(z)+\sigma_{j+\frac{1}{2}}(z)}{2} .
$$

Then the scheme (3.7)-(3.8) is well balance, i.e., $\frac{\mathrm{d}}{\mathrm{d} t} \bar{v}_{j}(t)=0$ for steady states of rest.

Proof. Consider a steady state at rest $w=$ Const., $u=0$. We notice that in the present case, $H_{j \pm \frac{1}{2}}^{A^{T}}=0$. For the discharge equation, using the discretized form of Leibniz's rule for differentiation under the integral sign,

$$
\Delta_{x} \int_{a(x)}^{b(x)} f(x, z) \mathrm{d} z=\frac{1}{2}\left(\int_{a_{L}}^{b_{L}}+\int_{a_{R}}^{b_{R}}\right) \Delta_{x} f(x, z) \mathrm{d} z+\int_{b_{L}}^{b_{R}} \bar{f}(z) \mathrm{d} z-\int_{a_{L}}^{a_{R}} \bar{f}(z) \mathrm{d} z,
$$

we observe that the flux difference as calculated in (3.24) amounts to

$$
\begin{aligned}
\frac{H_{j+\frac{1}{2}}^{Q}-H_{j-\frac{1}{2}}^{Q}=}{\Delta x} & \frac{1}{\Delta x} \frac{g}{2}\left(\int_{B_{j-\frac{1}{2}}}^{w_{j-\frac{1}{2}}}+\int_{B_{j+\frac{1}{2}}}^{w_{j+\frac{1}{2}}}\right) \Delta_{x}((w-z) \sigma)(z) \mathrm{d} z \\
& +\frac{1}{\Delta x} g \int_{w_{j-\frac{1}{2}}}^{w_{j+\frac{1}{2}}} \overline{(w-z) \sigma(x, z)} \mathrm{d} z-\frac{1}{\Delta x} g \int_{B_{j-\frac{1}{2}}}^{B_{j+\frac{1}{2}}} \overline{(w-z) \sigma(x, z)} \mathrm{d} z \\
= & \frac{1}{\Delta x} \frac{g}{2}\left(\int_{B_{j-\frac{1}{2}}}^{w_{j-\frac{1}{2}}}+\int_{B_{j+\frac{1}{2}}}^{w_{j+\frac{1}{2}}}\right)\left(w_{j}-z\right) \Delta \sigma_{j}(z) \mathrm{d} z-\frac{1}{\Delta x} g \int_{B_{j-\frac{1}{2}}}^{B_{j+\frac{1}{2}}}\left(w_{j}-z\right) \bar{\sigma}_{j}(z) \mathrm{d} z \\
= & \bar{S}_{j}(t) \text { for steady states of rest. }
\end{aligned}
$$




\section{Remark 3.2.}

1. The approximation (3.26) results from applying the trapezoidal rule in the $x$-direction, (3.27) follows from changing the direction of integration with the change of variables $z=B(x)$.

2. The integrals in the $z$-direction from the flux difference, $\left(H_{j+\frac{1}{2}}-H_{j-\frac{1}{2}}\right) / \Delta x$, and in (3.26)-(3.27) must be discretized consistently so as to ensure well balance. To this end, several options are available within the second order accuracy of the scheme, for instance, one could employ the composite trapezoidal or midpoint rules over the intervals $z \in\left[B_{j \pm \frac{1}{2}}, w_{j \pm \frac{1}{2}}\right]$ for both sets of integrals.

3. Quadrature formulae, however, are not the only or most convenient approach for the implementation of the scheme. For the results presented in Section 4 below, we chose to sample the channel geometry, $B_{j \pm \frac{1}{2}}$ and $\sigma_{j \pm \frac{1}{2}, k}$ at the points $\left(x_{j \pm \frac{1}{2}}, z_{k}\right)$ and we connect these values with piecewise linear functions (i.e., the cross-sections of the channel are piecewise trapezoidal). This approach allows us to calculate the integrals in the $z$-direction (and also those in (3.19)) exactly. Such discretization of the channel does, indeed, amount to applying the composite midpoint rule to evaluate those integrals.

\subsection{Evolution}

Once the interface values, the numerical fluxes and the average of the source term have been calculated, the ODE system (3.7) is integrated in time using the second order Strong Stability Preserving Runge-Kutta scheme [16],

$$
\begin{aligned}
v^{(1)} & =v^{(0)}+\Delta t C\left[v^{(0)}\right] \\
v^{(2)} & =\frac{1}{2} v^{(0)}+\frac{1}{2}\left(v^{(1)}+\Delta t C\left[v^{(1)}\right]\right) \\
\bar{v}(t+\Delta t) & :=v^{(2)}
\end{aligned}
$$

with the Runge-Kutta fluxes

$$
C[v(t)]=-\frac{H_{j+\frac{1}{2}}(v(t))-H_{j-\frac{1}{2}}(v(t))}{\Delta x}+\bar{S}_{j}(t)
$$

and $\bar{S}_{j}(t)$ calculated according to $(3.26)-(3.27)$. The time step $\Delta t$ is determined so as to satisfy the $C F L$ restriction

$$
\frac{\Delta t}{\Delta x} \leq \frac{1}{2 \max \left(a_{j-\frac{1}{2}} \max \left(\frac{A_{j-\frac{1}{2}}^{+}}{A_{j}}, 1\right), a_{j+\frac{1}{2}} \max \left(\frac{A_{j+\frac{1}{2}}^{-}}{A_{j}}, 1\right)\right)} .
$$

The following Proposition shows that this $C F L$ condition guarantees the positivity of the water height when the solution is evolved with forward Euler method:

Proposition 3.3. Consider the scheme (3.7)-(3.8) with the reconstruction algorithm described in Section 3.2 and the discretization of the source term (3.26)-(3.27). If the cell averages $\bar{A}^{T}(t)$ are such that

$$
\bar{w}_{j}(t) \geq \frac{B_{j-\frac{1}{2}}+B_{j+\frac{1}{2}}}{2} \quad \forall j
$$

or equivalently

$$
A_{j}^{T} \geq \int_{0}^{\frac{B_{j-\frac{1}{2}+B_{j+\frac{1}{2}}}^{2}}{{ }^{2}}} \bar{\sigma}_{j}(z) \mathrm{d} z
$$


where

$$
\bar{\sigma}(z):=\frac{\sigma\left(x_{j-\frac{1}{2}}, z\right)+\sigma\left(x_{j+\frac{1}{2}}, z\right)}{2},
$$

then the cell averages $\bar{A}^{T}(t+\Delta t)$ as evolved with forward Euler's method (3.29a) (i.e., $\bar{v}_{j}(t+\Delta t)=v^{(1)}$ in (3.29)) under the CFL limitation (3.31) with

$$
a_{j \pm \frac{1}{2}}=\max \left\{\left|u_{j \pm \frac{1}{2}}^{-}\right|+c_{j \pm \frac{1}{2}}^{-},\left|u_{j \pm \frac{1}{2}}^{+}\right|+c_{j \pm \frac{1}{2}}^{+}\right\}, \quad c_{j \pm \frac{1}{2}}^{ \pm}=\sqrt{\frac{A_{j \pm \frac{1}{2}}^{ \pm}}{\sigma_{j \pm \frac{1}{2}}^{T, \pm}}, \quad \text { and } \quad \sigma_{j \pm \frac{1}{2}}^{T, \pm}}=\sigma\left(x_{j \pm \frac{1}{2}}, w_{j \pm \frac{1}{2}}^{ \pm}\right),
$$

will yield

or equivalently

$$
\bar{A}_{j}^{T}(t+\Delta t) \geq \int_{0}^{\frac{B_{j-\frac{1}{2}+B_{j+\frac{1}{2}}}^{2}}{2}} \bar{\sigma}_{j}(z) \mathrm{d} z \quad \forall j
$$

$$
\bar{w}_{j}(t+\Delta t) \geq \frac{B_{j-\frac{1}{2}}+B_{j+\frac{1}{2}}}{2} \quad \forall j
$$

Proof. For $\lambda=\frac{\Delta t}{\Delta x}$, the updated cell average of $A^{T}$ satisfies

$$
\begin{aligned}
\bar{A}_{j}^{T}(t+\Delta t)= & \bar{A}_{j}^{T}(t)-\frac{\lambda}{2}\left[\left(Q_{j+\frac{1}{2}}^{+}+Q_{j+\frac{1}{2}}^{-}\right)-a_{j+\frac{1}{2}}\left(A_{j+\frac{1}{2}}^{T,+}-A_{j+\frac{1}{2}}^{T,-}\right)\right. \\
& \left.-\left(Q_{j-\frac{1}{2}}^{+}+Q_{j-\frac{1}{2}}^{-}\right)+a_{j-\frac{1}{2}}\left(A_{j-\frac{1}{2}}^{T,+}-A_{j-\frac{1}{2}}^{T,-}\right)\right] \\
= & \bar{A}_{j}^{T}(t)+\frac{\lambda}{2}\left[\left(a_{j+\frac{1}{2}}-u_{j+\frac{1}{2}}^{+}\right) A_{j+\frac{1}{2}}^{+}+\left(a_{j-\frac{1}{2}}+u_{j-\frac{1}{2}}^{-}\right) A_{j-\frac{1}{2}}^{-}\right] \\
& -\frac{\lambda}{2}\left[\left(a_{j+\frac{1}{2}}+u_{j+\frac{1}{2}}^{-}\right) A_{j+\frac{1}{2}}^{-}+\left(a_{j-\frac{1}{2}}-u_{j-\frac{1}{2}}^{+}\right) A_{j-\frac{1}{2}}^{+}\right] \\
\geq & \bar{A}_{j}^{T}(t)-\frac{\lambda}{2}\left[\left(a_{j+\frac{1}{2}}+u_{j+\frac{1}{2}}^{-}\right) A_{j+\frac{1}{2}}^{-}+\left(a_{j-\frac{1}{2}}-u_{j-\frac{1}{2}}^{+}\right) A_{j-\frac{1}{2}}^{+}\right],
\end{aligned}
$$

and applying the $C F L$ restriction (3.31), we obtain

$$
\bar{A}_{j}^{T}(t+\Delta t) \geq \bar{A}_{j}^{T}-\lambda\left[a_{j+\frac{1}{2}} A_{j+\frac{1}{2}}^{-}+a_{j-\frac{1}{2}} A_{j-\frac{1}{2}}^{+}\right] \geq \bar{A}_{j}^{T}(t)-\bar{A}_{j}(t)=\int_{0}^{\frac{B_{j-\frac{1}{2}}+B_{j+\frac{1}{2}}}{2}} \bar{\sigma}_{j}(z) \mathrm{d} z,
$$

which concludes the proof.

Remark 3.4. The second-order SSP Runge-Kutta scheme (3.29) consists of a convex combination of successive forward Euler steps, therefore, for scalar equations, proving that a particular property of the semi-discrete formulation (3.7)-(3.8) holds when the system of ODEs is evolved with forward Euler's method will suffice to prove such property holds when higher-order SSP Runge-Kutta schemes are employed, [16]. For the shallowwater system discussed here, however, positivity can only be proven for the first-order Euler's method since the techniques suggested to ensure positivity at the cell interfaces and the regularization of the velocity, (3.20), may dictate a smaller value of $\Delta t$ for subsequent stages of higher order evolution routine. The numerical results presented below, however, suggest that, in most cases, the scheme remains stable under the $C F L$ restriction (3.31) when the full second-order Runge-Kutta scheme (3.29) is employed. Only in cases where the regularization of the velocity needs to be applied some oscillations may arise, requiring a smaller value of $\Delta t$ to ensure stability, regardless of the ODE solver used for the time integration. We refer the reader to [7] for dry bed modification algorithm that ensures positivity without any impact on the global time step. 

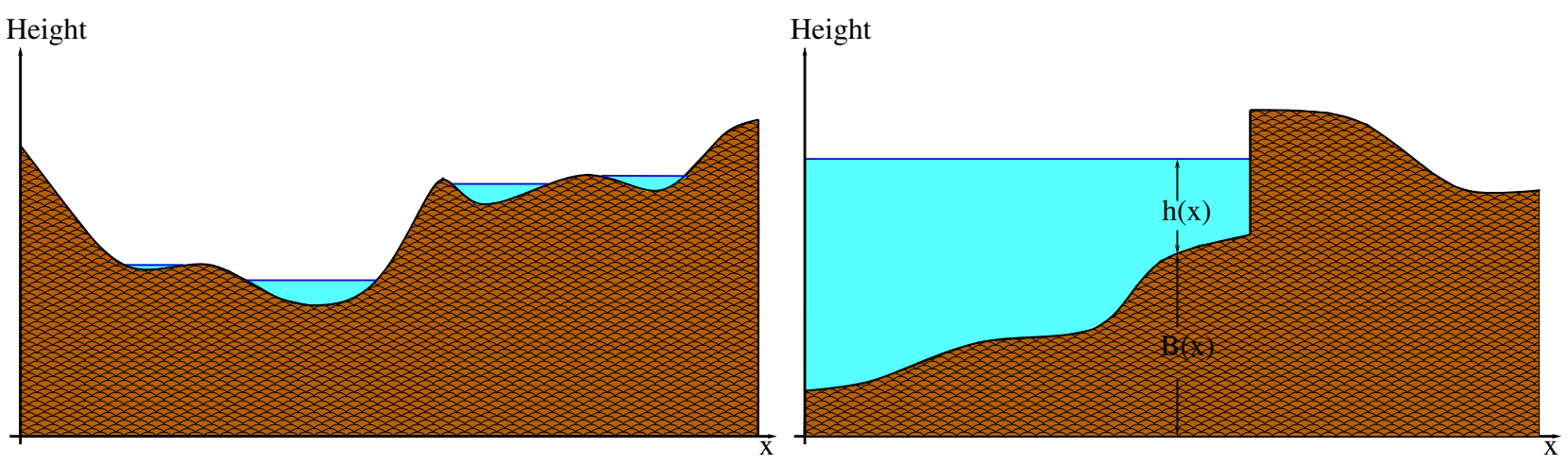

FiguRE 3. Stationary solutions involving wet and dry states in a topography with several bumps (left) and in a topography with jumps (right).
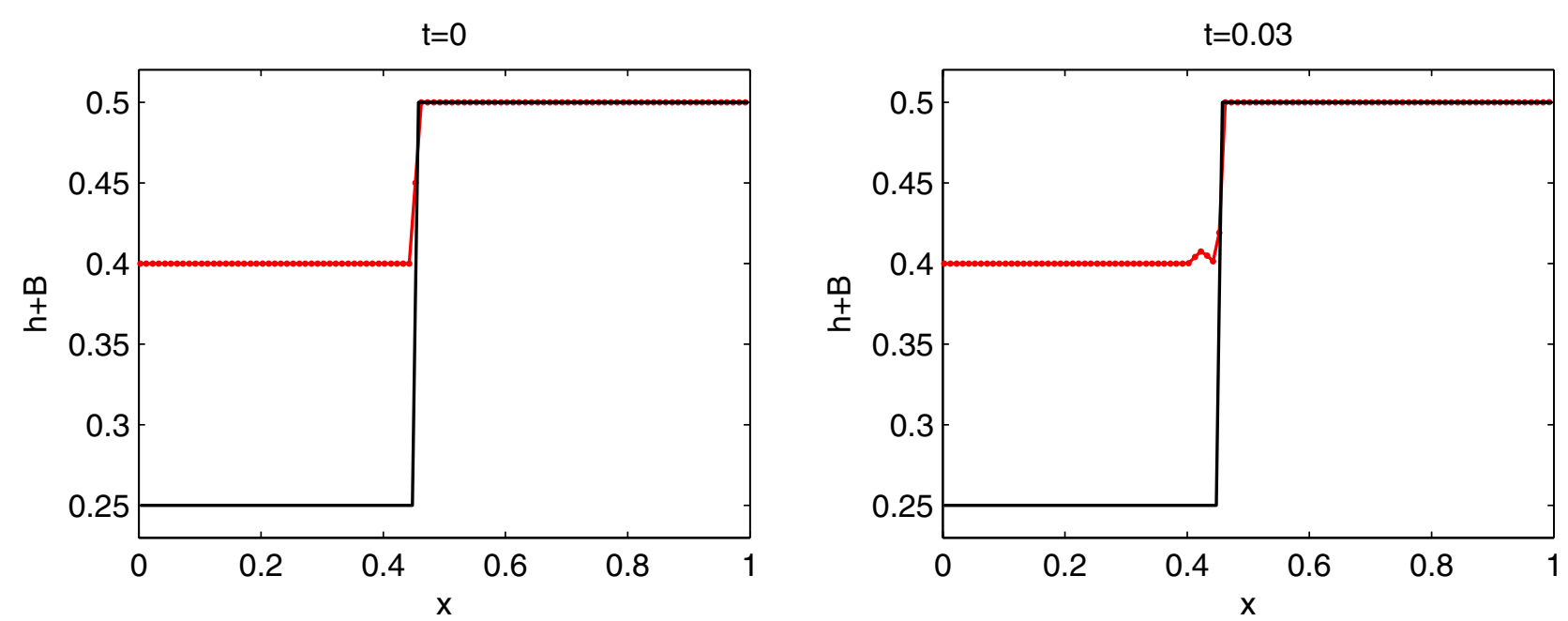

FIgURE 4. Numerical solution of a steady state at rest with wet-dry states. Spurious oscillations near wet-dry states are observed. The topography is given by the black solid line and the total height by the red dotted line.

\subsection{Treatment of wet-dry states}

Coastal flows, flooding simulations, and other geophysical problems often lead to solutions involving wet and dry states. For example, stationary wet-dry fronts like those of Figure 3 - where $w$ is constant in the wet region and $w=B$ in the dry region - may occur if the bottom topography contains bumps or jump discontinuities, and dam break problems may lead to non-stationary flows with wet and dry fronts like the one illustrated in Figure 5, where the dry areas will be flooded and the solution will not remain stationary. The additional difficulties that these wet-dry fronts pose - like the preservation of positivity in dry cells - have been studied in $[4,6]$.

The well-balance property of Section 3.3 allows our scheme to recognize and preserve steady states of rest where the total height $w=h+B$ is constant and $u=0$, but it does not take into account possible dry areas. As a result, numerical spurious oscillations may be generated near the wet-dry interfaces as the well-balance property does not guarantee the preservation of the wet-dry stationary solutions. Figure 4 shows the evolution 


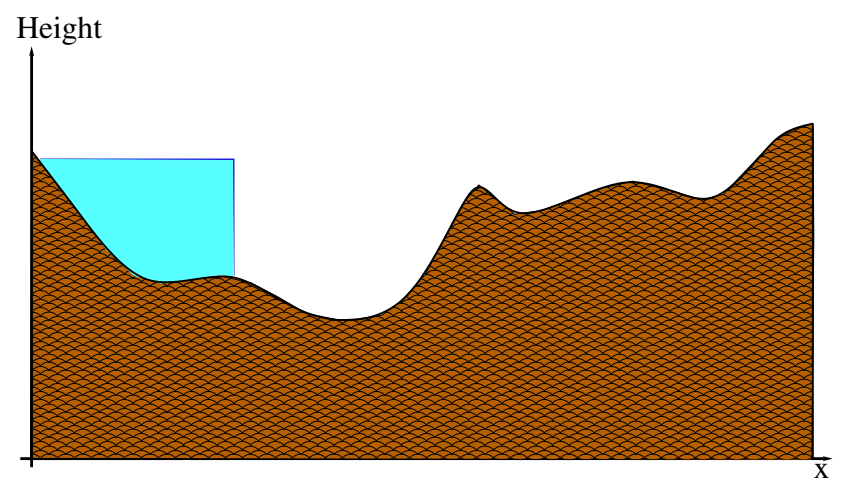

FiguRE 5. Example of a wet-dry state with constant height in wet areas, and not stationary.

in time of a wet-dry steady state at rest at time $t=0$ (left) and $t=0.03$ (right) in a channel with straight walls $(\sigma \equiv 1)$ and piece-wise constant topography. The numerical approximation does not stay stationary and noise generated near the jump starts propagating westwards. A modification of the scheme will be needed to enable it to preserve wet-dry steady states.

\subsubsection{An improved well-balance property}

Following [6], we describe a wet-dry state by splitting the domain into two non overlapping parts: $X_{1}$ (wet area) and $X_{2}$ (dry area). The interface points are $I^{W D}=X_{1} \cap X_{2}$. A wet-dry state is called at rest provided that

(a) The velocity $u$ is zero everywhere

(b) $w$ is constant on each connected component of $X_{1}$

(c) $w=B$ on $X_{2}$

(d) For each $x \in I^{W D}$, there exists $\epsilon>0$ such that $w=w_{0}$ is constant on $(x-\epsilon, x+\epsilon) \cap X_{1}$ and that constant $w_{0}$ satisfies $w_{0}<B$ on $(x-\epsilon, x+\epsilon) \cap X_{2}$. This prevents the wet state from inundating the dry area on the other side of the interface, as in Figure 5.

This definition describes wet-dry states like those in Figure 3 and leads us to consider the following reference scenario to devise an improved well-balance discretization that preserves steady states in (and near) dry cells: we assume that a wet-dry interface occurs at cell $I_{j}$ and the dry area is located to the right of the interface (the derivation would be identical but with indeces $j+\frac{1}{2}$ and $j-\frac{1}{2}$ exchanged), and the cell average of $w$ in cell $I_{j}$ is set to $\bar{w}_{j}=\frac{w_{j-\frac{1}{2}}+B_{j+\frac{1}{2}}}{2}<B_{j+\frac{1}{2}}$, where the interface values of $w$ satisfy $w_{j+1 / 2}=B_{j+1 / 2}$ and $w_{j-1 / 2}=w_{w e t}<B_{j+1 / 2}$, preventing the flow from moving and inundating the right side of cell $I_{j}$. Figure 6 illustrates this case.

In [6] preservation of steady-states even in the presence of dry areas is achieved by modifying the reconstruction of water's depth in almost dry cells, here we take a different approach consisting in a modification of the discretization of the averaged cross-sectional hydrostatic pressure in equation (2.3). The following proposition guarantees preservation of steady states at rest, even if dry areas are present. It is based on a modified numerical flux at cell $j$. This modification is done near possible interfaces, which can be tracked at points where

$$
\begin{array}{r}
\min \left(B_{j-1 / 2}, B_{j+1 / 2}\right)<\bar{w}_{j}<\max \left(B_{j-1 / 2}, B_{j+1 / 2}\right) \text { and } \bar{w}_{j+1}>\max \left(B_{j+1 / 2}, B_{j+3 / 2}\right) \\
\min \left(B_{j-1 / 2}, B_{j+1 / 2}\right)<\bar{w}_{j}<\max \left(B_{j-1 / 2}, B_{j+1 / 2}\right) \text { and } \bar{w}_{j-1}>\max \left(B_{j-3 / 2}, B_{j-1 / 2}\right) .
\end{array}
$$




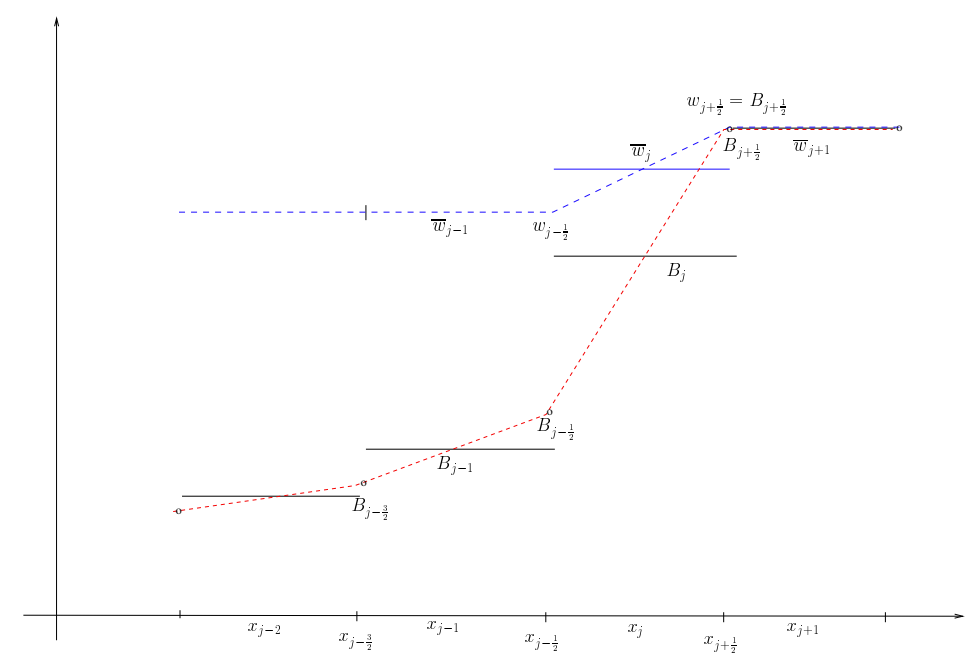

Figure 6. Flow profile for a Wet-Dry flow. The linear reconstruction of the total water elevation $w$ across the wet-dry cell matches both states at the cell interfaces, $w_{j \pm \frac{1}{2}}$, while its cell average, $\bar{w}_{j}$, remains below the wet state, preventing inundation of the dry state.

Proposition 3.5. The numerical scheme (3.29), (3.30), (3.8) with modified numerical flux at cells $j$ satisfying equation (3.33), given by the following averaged cross-sectional hydrostatic pressure

$$
\begin{gathered}
I_{1, j \pm \frac{1}{2}}^{W D}=\frac{g}{2 \Delta x}\left[\int_{B_{j \pm \frac{1}{2}}}^{w_{j \mp \frac{1}{2}}^{ \pm}}\left(\bar{w}_{j}-z\right) \sigma_{j \pm \frac{1}{2}}(z) \mathrm{d} z+\int_{B_{j \pm \frac{1}{2}}}^{w_{j \pm \frac{1}{2}}^{\mp}}\left(\bar{w}_{j}-z\right) \sigma_{j \pm \frac{1}{2}}(z) \mathrm{d} z\right] \\
H_{j \pm \frac{1}{2}}=\left(\begin{array}{c}
Q_{j \pm 1 / 2} \\
\frac{Q_{j \pm 1 / 2}^{2}}{A_{j \pm 1 / 2}^{T}-\int_{0}^{B} \sigma_{j \pm 1 / 2} \sigma_{j \pm 1 / 2}(z) \mathrm{d} z}+I_{1, j \pm \frac{1}{2}}^{W D}
\end{array}\right),
\end{gathered}
$$

and

$$
a_{j \pm \frac{1}{2}}^{W D}=\max \left(\max \left(u_{j \pm \frac{1}{2}}^{+}, u_{j \pm \frac{1}{2}}^{-}\right), \min \left(\left|u_{j \pm \frac{1}{2}}^{-}\right|+c_{j \pm \frac{1}{2}}^{-},\left|u_{j \pm \frac{1}{2}}^{+}\right|+c_{j \pm \frac{1}{2}}^{+}\right)\right)
$$

preserves wet-dry steady states at rest.

Proof. Assume for simplicity that we only have one wet-dry interface, located at cell $j$ (for some $j$ ) as in Figure 6 in the case above. Then $w_{j-\frac{1}{2}}^{+}=w_{w e t}$, where $w_{w e t}$ is the wet state, and $w_{j+\frac{1}{2}}^{-}=B_{j+\frac{1}{2}}$. The diffusion coefficients $a_{j \pm \frac{1}{2}}$ are an estimate to the maximum wave speed, which is needed for stability. However, the estimate $a_{j \pm \frac{1}{2}}$ in equation (3.32) does not recognize wet-dry steady states since it does not vanish at the wetdry interface. The modified estimate (3.36) vanishes at a wet-dry interface, while still satisfying $a_{j \pm \frac{1}{2}} \geq\left|u_{j \pm \frac{1}{2}}\right|$, which is needed for positivity.

Since $w_{j-\frac{1}{2}}^{+}=w_{j-\frac{1}{2}}^{-}=w_{w e t}, w_{j+\frac{1}{2}}^{+}=w_{j+\frac{1}{2}}^{-}=B_{j+\frac{1}{2}}$, and $u=0$, the first entry in the numerical fluxes $H_{j \pm \frac{1}{2}}^{A^{T}}$ vanishes. And since $u=0$ and $c_{j+\frac{1}{2}}^{ \pm}=0, a_{j \pm \frac{1}{2}}^{W D}=0$, and the second component of the numerical flux at the left 
cell interface reads

$$
\begin{aligned}
H_{j-\frac{1}{2}}^{Q} & =I_{1, j-\frac{1}{2}}^{W D}=\frac{g}{2}\left[\int_{B_{j-\frac{1}{2}}}^{w_{j+\frac{1}{2}}^{-}}\left(\bar{w}_{j}-z\right) \sigma_{j-\frac{1}{2}}(z) \mathrm{d} z+\int_{B_{j-\frac{1}{2}}}^{w_{j-\frac{1}{2}}^{+}}\left(\bar{w}_{j}-z\right) \sigma_{j-\frac{1}{2}}(z) \mathrm{d} z\right] \\
& =\frac{g}{2}\left[\int_{B_{j-\frac{1}{2}}}^{B_{j+\frac{1}{2}}}\left(\bar{w}_{j}-z\right) \sigma_{j-\frac{1}{2}}(z) \mathrm{d} z+\int_{B_{j-\frac{1}{2}}}^{w_{w e t}}\left(\bar{w}_{j}-z\right) \sigma_{j-\frac{1}{2}}(z) \mathrm{d} z\right],
\end{aligned}
$$

and at the right cell interface

$$
H_{j+\frac{1}{2}}^{Q}=I_{1, j+\frac{1}{2}}^{W D}=\frac{g}{2}\left[\int_{B_{j+\frac{1}{2}}}^{w_{j-\frac{1}{2}}^{+}}\left(\bar{w}_{j}-z\right) \sigma_{j+\frac{1}{2}}(z) \mathrm{d} z+\int_{B_{j+\frac{1}{2}}}^{w_{j+\frac{1}{2}}^{-}}\left(\bar{w}_{j}-z\right) \sigma_{j+\frac{1}{2}}(z) \mathrm{d} z\right]=\frac{g}{2} \int_{B_{j+\frac{1}{2}}}^{w_{w e t}}\left(\bar{w}_{j}-z\right) \sigma_{j+\frac{1}{2}}(z) \mathrm{d} z
$$

Therefore, the flux difference amounts to

$$
\frac{H_{j+\frac{1}{2}}^{Q}-H_{j-\frac{1}{2}}^{Q}}{\Delta x}=\frac{g}{2 \Delta x}\left[\int_{B_{j+\frac{1}{2}}}^{w_{w e t}}\left(\bar{w}_{j}-z\right) \sigma_{j+\frac{1}{2}}(z) \mathrm{d} z-\int_{B_{j-\frac{1}{2}}}^{B_{j+\frac{1}{2}}}\left(\bar{w}_{j}-z\right) \sigma_{j-\frac{1}{2}}(z) \mathrm{d} z-\int_{B_{j-\frac{1}{2}}}^{w_{w e t}}\left(\bar{w}_{j}-z\right) \sigma_{j-\frac{1}{2}}(z) \mathrm{d} z\right] .
$$

On the other hand, the cell average of the source term is given by

$$
\begin{aligned}
\bar{S}_{j}(t)= & \frac{g}{2 \Delta x}\left(\int_{B_{j-\frac{1}{2}}}^{w_{j-\frac{1}{2}}^{+}}+\int_{B_{j+\frac{1}{2}}}^{w_{j+\frac{1}{2}}^{-}}\right)\left(\bar{w}_{j}-z\right) \Delta \sigma_{j}(z) \mathrm{d} z-\frac{g}{\Delta x} \int_{B_{j-\frac{1}{2}}}^{B_{j+\frac{1}{2}}}\left(\bar{w}_{j}-z\right) \bar{\sigma}_{j}(z) \mathrm{d} z \\
= & \frac{g}{2 \Delta x} \int_{B_{j-\frac{1}{2}}}^{w_{j-\frac{1}{2}}^{+}}\left(\bar{w}_{j}-z\right) \Delta \sigma_{j}(z) \mathrm{d} z-\frac{g}{\Delta x} \int_{B_{j-\frac{1}{2}}}^{B_{j+\frac{1}{2}}}\left(\bar{w}_{j}-z\right) \bar{\sigma}_{j}(z) \mathrm{d} z \\
= & \frac{g}{2 \Delta x} \int_{B_{j-\frac{1}{2}}}^{w_{j-\frac{1}{2}}^{+}}\left(\bar{w}_{j}-z\right) \sigma_{j+\frac{1}{2}}(z) \mathrm{d} z-\frac{g}{2 \Delta x} \int_{B_{j-\frac{1}{2}}}^{w_{j-\frac{1}{2}}^{+}}\left(\bar{w}_{j}-z\right) \sigma_{j-\frac{1}{2}}(z) \mathrm{d} z \\
& +\frac{g}{2 \Delta x} \int_{B_{j+\frac{1}{2}}}^{B_{j-\frac{1}{2}}}\left(\bar{w}_{j}-z\right) \sigma_{j+\frac{1}{2}}(z) \mathrm{d} z-\frac{g}{2 \Delta x} \int_{B_{j-\frac{1}{2}}}^{B_{j+\frac{1}{2}}}\left(\bar{w}_{j}-z\right) \sigma_{j-\frac{1}{2}}(z) \mathrm{d} z \\
= & \frac{H_{j+\frac{1}{2}}^{Q}-H_{j-\frac{1}{2}}^{Q}}{\Delta x}
\end{aligned}
$$

which concludes the proof.

Note that this modification affects the second component of the numerical flux, and it does not affect the positivity preserving property. This approach has been tested in the numerical results in the next section.

\section{Numerical Results}

In this section we present the numerical solution of several prototype problems aimed at demonstrating the properties of our central scheme and its ability to capture non-trivial steady flows. We begin by validating the well balance property of the scheme and its behavior under small perturbations from the trivial steadystate, (2.6). These include perturbations from piecewise trivial steady states so as to test the ability of the 
scheme to identify and resolve correctly wet-dry states. We also study the convergence of solutions evolved with the central scheme to non-trivial steady state solutions by comparing them to exact steady flows. To conclude, we test the positivity preserving property by considering first an oscillating mass of water that changes parts of the channel from dry to wet and wet to dry as time evolves, and with a dam break problem where water is allowed to flow out of the channel onto a dry bed.

The flows are calculated along channels with arbitrary geometry and bottom topography. We consider the geometry described by the width functions

$$
\begin{aligned}
\sigma_{1}(x, z)=1 & +\frac{3}{4} \cos (\pi x)-\frac{1}{4} \chi_{[0.4,0.6]}(x)(\cos (\pi(x-1 / 2) / 0.1)+1) \\
& +\sqrt{z}\left(1-\frac{1}{4} \chi_{[0.1,0.7]}(x)(\cos (\pi(x-0.4) / 0.3)+1)\right) \\
& -1.2 \chi_{[0,1]}\left(\frac{(x-0.3)^{2}+(z-1.4)^{2}}{r_{1}^{2}}\right) \cos \left(\sqrt{\frac{(x-0.3)^{2}+(z-1.4)^{2}}{r_{1}^{2}}} \frac{\pi}{2}\right) \\
& -1.2 \chi_{[0,1]}\left(\frac{(x-0.75)^{2}+(z-1.4)^{2}}{r_{2}^{2}}\right) \cos \left(\sqrt{\frac{(x-0.75)^{2}+(z-1.4)^{2}}{r_{2}^{2}}} \frac{\pi}{2}\right),
\end{aligned}
$$

where $r_{1}=0.28, r_{2}=0.2$. Here $\chi_{[a, b]}$ is the characteristic function on any interval $[a, b]$, and

$$
\sigma_{2}(x, z)=\frac{1}{2}\left(1+\sqrt{z}\left(1-\frac{1}{4}(\cos (\pi(x-0.6) / 0.2)+1) \chi_{[0.4,0.8]}(x)\right)\right) .
$$

These width functions are combined with different bottom topographies so as to render geometries suitable to create the flows that are most challenging for the properties of the scheme to be tested on each case.

Unless otherwise stated, for the results presented below the value of the acceleration of gravity is taken as $g=9.81$ and the time step, $\Delta t$, satisfies the $C F L$ restriction

$$
\frac{\Delta t}{\Delta x} \leq \frac{\tau}{\max \left(a_{j-\frac{1}{2}} \max \left(\frac{A_{j-\frac{1}{2}}^{+}}{A_{j}}, 1\right), a_{j+\frac{1}{2}} \max \left(\frac{A_{j+\frac{1}{2}}^{-}}{A_{j}}, 1\right)\right)}, \quad \tau<1 .
$$

where $a_{j \pm \frac{1}{2}}$ stands for the estimates of the maximum speeds of propagation at the cell interfaces, calculated as (3.32) (or (3.36) where appropriate). We shall note here that while the proof of proposition 3.3 requires $\tau<\frac{1}{2}$, most of the numerical experiments below were computed with values $\frac{1}{2}<\tau<1$. The same flows simulated with a more restrictive $C F L$ number, $\tau<\frac{1}{2}$, did not yield substantially better results. In Section 4.1 we set $\tau=0.45$ and study the convergence of the scheme by progressively reducing the grid side. For the perturbation of a steady state of rest in Section 4.2 we use $\tau=0.45$ to reduce oscillations caused by the narrowest areas of the channel, and the oscillating lake simulation in Section 4.5 was computed with $\tau=0.6$ and $\alpha=1$ (in Eq. (3.14)) also to prevent the onset of oscillations. All other flows were calculated with $\tau=0.9$ and $\alpha=1.5$.

\subsection{Accuracy of the scheme}

In this section we consider two flows in order to study the convergence of the scheme over a successively refined grid. The first example consist of a trapezoidal channel with smooth initial conditions that remain smooth for a short time. The results obtained over the successively thinner grids validate the second order of the scheme for smooth flows. In the second example, as one would expect, the observed order of accuracy is reduced as shock waves develop within a flow containing wet-dry fronts. 

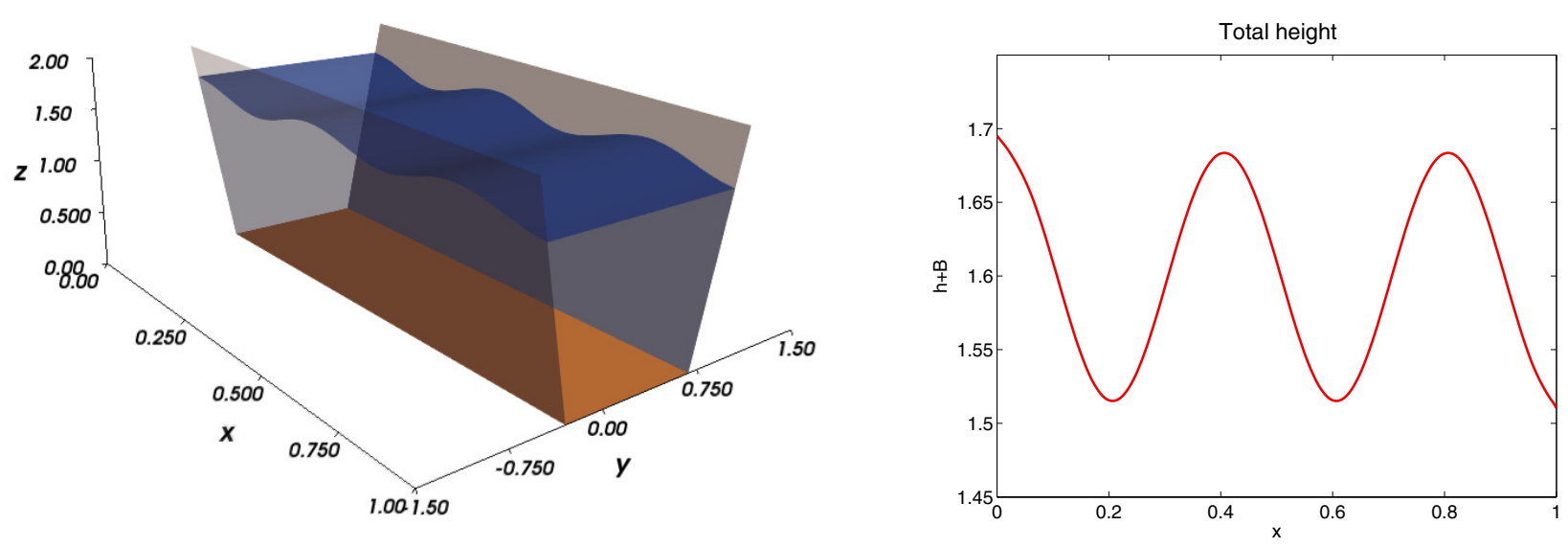

Figure 7 . Solution at time $t=0.01$ for a trapezoidal channel with initial conditions given in equation (4.5).

\subsubsection{Accuracy in smooth regions}

Complicated geometries may influence the presence of shock waves very quickly. For simplicity, and in order to guarantee the absence of shock waves in a short period of time, we consider a trapezoidal geometry

$$
\sigma(x, y)=1+0.3 y
$$

and a flat topography $B=0$. Initially the total height and the velocity are given by

$$
w(x, t=0)=1.7+0.1\left(\cos \left(\frac{\pi(x-0.4)}{0.2}\right)-1\right), \quad u(x, t=0)=1 .
$$

Assuming free boundary conditions, Figure 7 shows the total height (in $2 \mathrm{D}$ and 3D) at the final time $T=0.01$. No shock waves are observed, and second order accuracy is expected. We approximate the exact solution at time $T=0.01$ with a fine grid $(N=10240)$. In order to study convergence, we compute the $L^{1}$ error for $\Delta x=1 / N$, $N=20,40,80,160,320,640,1280,2560$ and 5120, respectively. The $L^{1}$ error and the corresponding ratio with $\Delta x^{2}$ are shown in Table 1. In addition, Figure 8 shows the corresponding log-log plot of the $L^{1}$ error, and two lines with slopes 1 and 2 for comparison. The results confirm the second order accuracy of the scheme.

\subsubsection{Accuracy near wet-dry states}

Having demonstrated the second order accuracy of the scheme in smooth regions, in this test we consider an oscillating lake like that in [4]. In general one can expect the accuracy of the scheme to be reduced to first order in the presence of shock waves. As a separate issue, the regularization (3.20) needed near dry areas introduces errors in the computations. As a result, the accuracy of the scheme near wet-dry states could be drastically reduced in the oscillating lake. An analysis of the accuracy follows.

The same trapezoidal geometry from equation (4.4) is considered. However, the topography is now given by

$$
B(x)=\frac{1}{4}(1-\cos (2 \pi(x-1 / 2))) .
$$

The initial conditions are given by

$$
\omega(x, t=0)=5 \times 10^{-4}+\max (B(x), 0.25+0.2 x), u=0 .
$$


TABLE 1. Second order accuracy in smooth regions.

\begin{tabular}{l|c|c}
\hline Number of grid points & $L^{1}$ Error & $L^{1}$ Error $/ \Delta x^{2}$ \\
\hline 20 & $2.7 \times 10^{-3}$ & 1.0 \\
40 & $1.5 \times 10^{-3}$ & 2.4 \\
80 & $5.6 \times 10^{-4}$ & 3.5 \\
160 & $1.1 \times 10^{-4}$ & 2.8 \\
320 & $2.9 \times 10^{-5}$ & 3.0 \\
640 & $7.4 \times 10^{-6}$ & 3.0 \\
1280 & $1.8 \times 10^{-6}$ & 3.1 \\
2560 & $4.4 \times 10^{-7}$ & 3.1 \\
5120 & $9.1 \times 10^{-8}$ & 3.1 \\
\hline
\end{tabular}

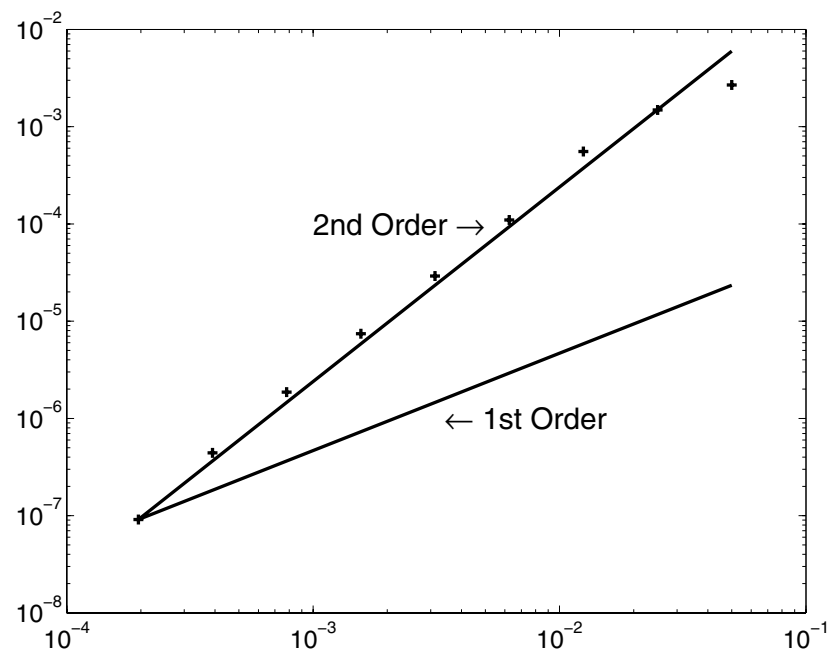

FIgURE 8. Log-log plot of $L^{1}$ error as a function of $\Delta x$ (dots) for the smooth flow with initial conditions (4.5). The two solid lines indicate first and second order accuracy.

The initial state is an oscillating lake as in [4], and can be seen in Figure 9 (left, dashed blue line). It corresponds to a lake with an inclined surface in the middle of the domain, and dry states otherwise. The evolution of the surface consist then of oscillations. At time $T=0.4$, the oscillating lake has completed half of an oscillations, as seen in Figure 9 (left, red solid line). Clear discontinuities can be identified in the velocity field (right plot), decreasing the accuracy of the scheme. The velocity discontinuities are located near the wetdry states, where the velocity regularization is needed. This process introduces further errors in the numerical results. Figure 10 shows the log-log plot of the $L^{1}$ error norm. The accuracy has been reduced to order 0.6 approximately.

\subsection{Perturbations of steady state of rest}

In this test, the geometry of the channel is given by the width (4.1) and a bottom topography is given by a spline of degree three with nodes at $(-0.1,1.4),(0.3,0.7),(0.6,0.6),(0.7,1),(0.8,0.7),(0.9,1.1)$, and $(1.1,1.3)$. Figure 11 shows a $3 \mathrm{D}$ view of the channel and the vertical profile of the topography. For the convenience of the reader, details on the computation of cubic spline interpolation is included in Appendix B. 

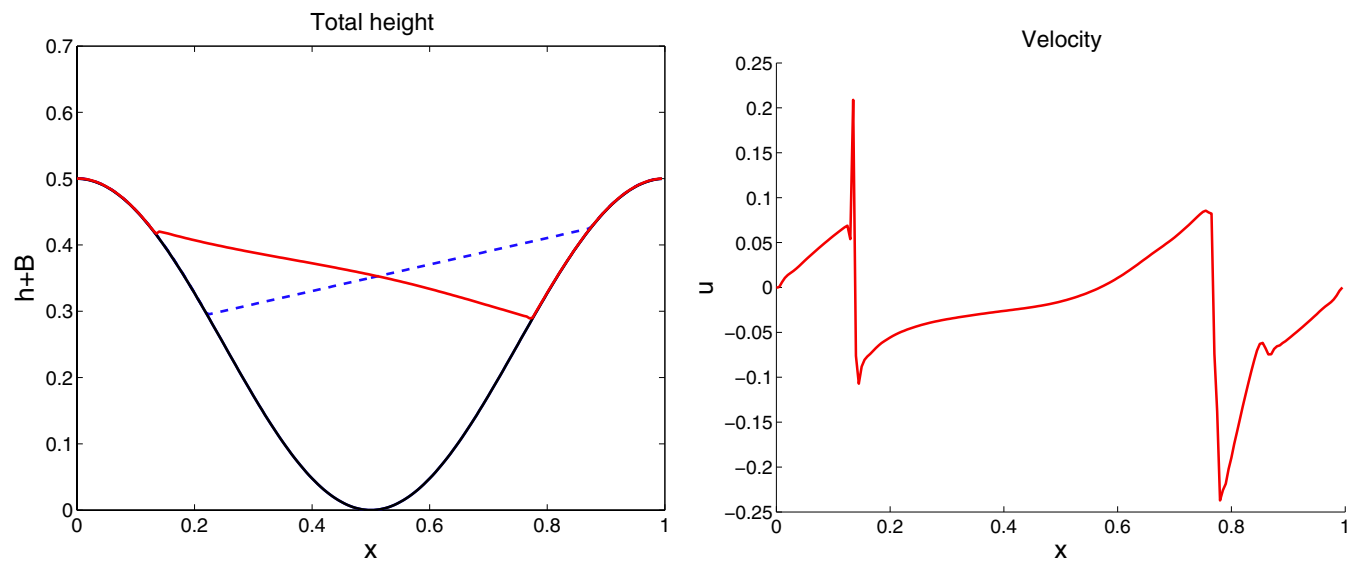

Figure 9. Solution at time $T=0.4$ for a trapezoidal channel with topography, and initial conditions given by (4.6) and (4.7). Dashed line denotes the initial condition, and solid line is the numerical solution at time $t=0.4$.

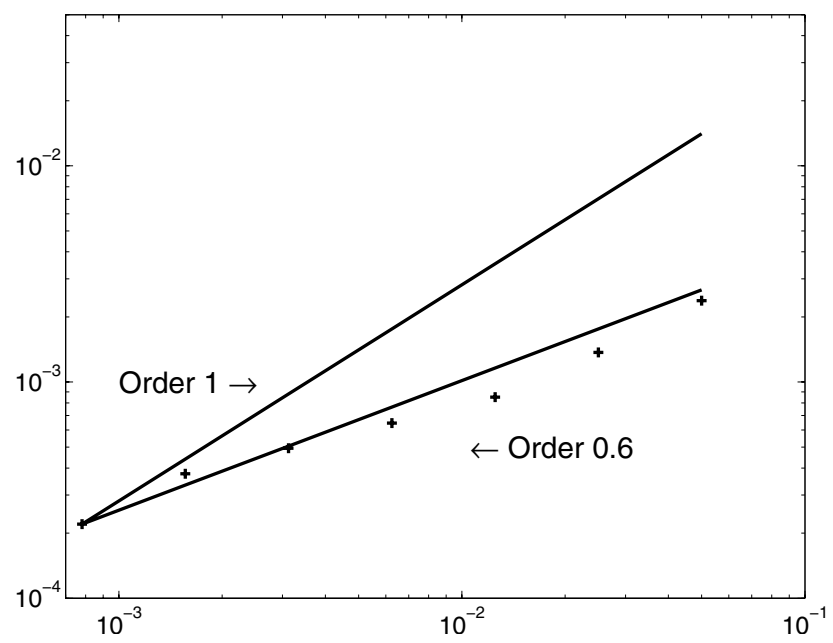

Figure 10. Log-log plot of $L^{1}$ error as a function of $\Delta x$ (dots) for the non-smooth flow with initial conditions (4.7). The two solid lines indicate order of accuraccy 1 and 0.6.

The proposed numerical scheme preserves steady states at rest by construction. It has been shown in related works $(e . g .,[14,17,31])$ that recognizing steady states at rest is enough to enable the scheme to recognize and compute near steady state solutions accurately. We begin testing our numerical scheme with the evolution of a perturbation from a steady state. These perturbation should propagate in both directions. Once the perturbation leaves the free boundary, only the flat states should remain in our computational domain, and the flow should converge to the trivial steady state (2.6).

The initial height is $w=1.45$ and a perturbation of size $\epsilon=10^{-2}$ is applied on the interval $[0.1,0.15]$. The flow is initially at rest. The topography and geometry are non-trivial at the boundaries which requires us to specify the values of the total height, $w$ and the velocity $u$, at both boundaries so as to implement free flow boundaries. 


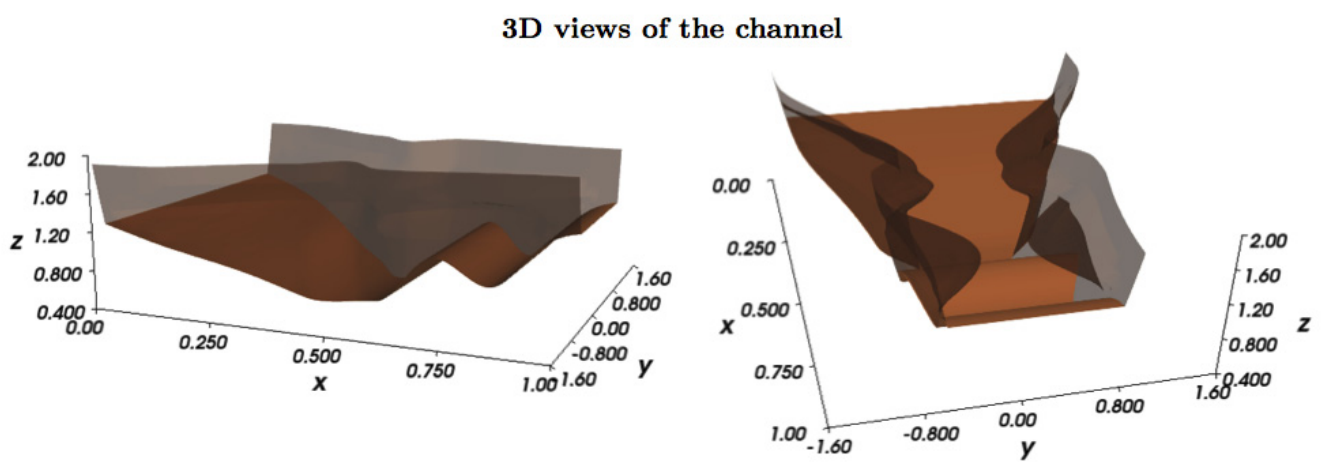

Bottom topography

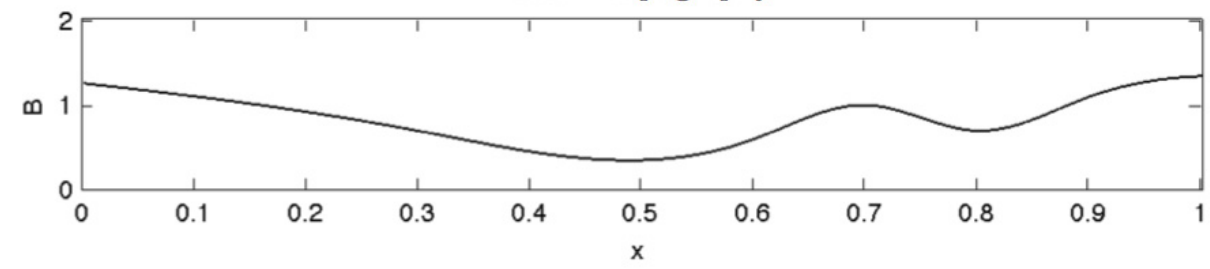

Figure 11. 3D-view of the channel (top) and bottom topography (bottom).

Figure 12 shows the evolution of the perturbation at times $t=0,0.02,0.2,2$. We compare the numerical results obtained by the present scheme (dotted line) to the numerical solution obtained using the upwind Roetype scheme (solid line) in [17]. The comparison shows a good agreement between the two schemes. Due to the non-trivial topography and general geometry of the channel's walls, a curved profile is developed as the perturbation jumps over the topography and the width of the channels changes at different heights. After the perturbation leaves the domain, we observe that an unperturbed steady state is recovered.

\subsection{Test on wet-dry states}

Positivity and preservation of wet-dry states are two properties achieved by this scheme. In this test we will verify the properties proved in propositions 3.3 and 3.5 in a discontinuous topography with a dry state on one side and a flat wet state on the other. The topography is a piece-wise spline. To obtain it, we first consider an spline of degree three with nodes $(0,0.3),(0.05,0.3),(0.1,0.2),(0.15,0.5),(0.3,0.4),(0.4,0.6)$, and $(0.75,0.6)$ (see Appendix B). Then the spline is rescaled by a factor of 0.5 for $x \leq 0.53$ and of 1.3 for $x \geq 0.53$. Finally the topography is redefined to be flat near the two boundaries: 0.12 for $x \leq 0.1$ and 0.8 for $x \geq 0.7$. A $3 \mathrm{D}$-view of the channel is shown Figure 13, the topography is shown in light brown and the side walls in gray.

A perturbation to the stationary state is applied to the left of the discontinuity in the topography. The perturbation propagates and hits the "jump" in the topography. If the perturbation is small enough, it is expected to reflect back without transmitting any wave to the right. When the perturbation is large, however, part of the wave reflects back and part is transmitted, inundating the right side and leaving the boundary through the right. We test both cases next.

\subsubsection{Small perturbation}

We first consider a perturbation of size $\epsilon=0.1$ to the left of the wet stationary state in the example above. Figure 14 shows the 2D (top) and 3D (bottom) views of the evolution in the perturbation. As observed above, the wave reflects back after hitting the "shore". 
Total height at $\mathrm{t}=0$

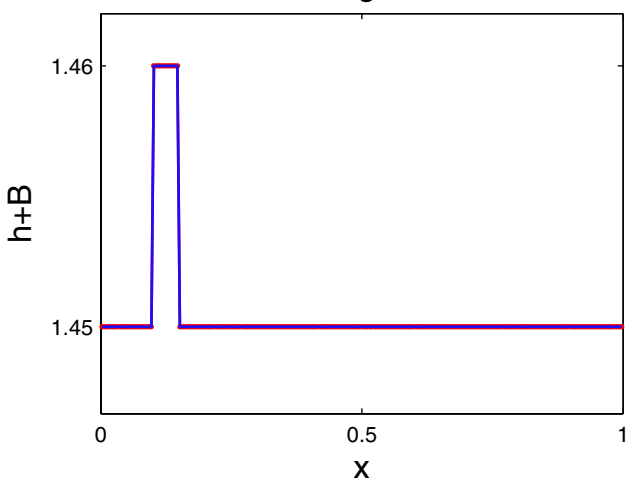

Total height at $\mathrm{t}=0.2$

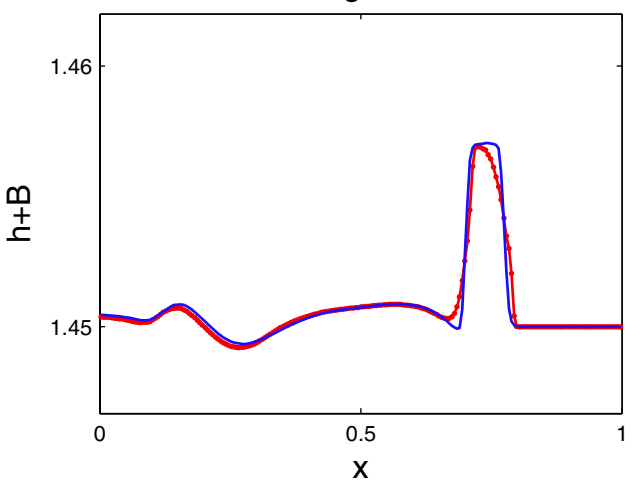

Total height at $\mathrm{t}=0.025$

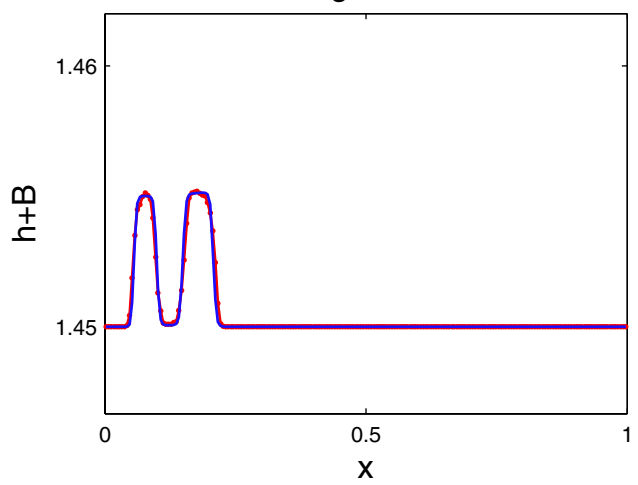

Total height at $\mathrm{t}=2$

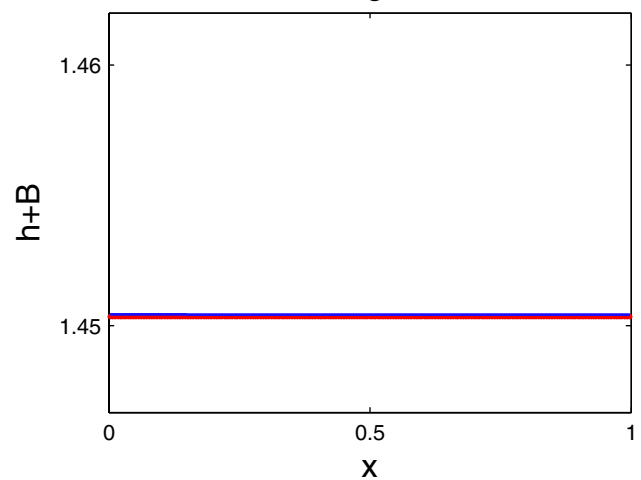

Figure 12. Perturbation of steady state of rest at $t=0,0.025,0.2,2$. The numerical results obtained with a Roe-type upwind scheme (solid line) and with the present scheme (dotted line) are compared, showing good agreement.

\subsubsection{Large perturbation}

A perturbation of size $\epsilon=0.3$ is applied on the left wet stationary state in the example above. Figure 15 shows the 2D (top) and 3D (bottom) views of the evolution in the perturbation. We observe the wave partially reflecting back and partially transmitting the discontinuity, eventually leaving through the right boundary.

\subsection{Convergence to Steady States}

As pointed out in Section 2.2, smooth steady-state flows are characterized by their constant discharge, $Q$, and energy, $E$. These steady or near steady flows are quite common in nature. In $[11,26,33]$ numerical schemes that preserve general steady-states and not just at rest have been constructed. In nature only near steady states are common, and in [17] it was shown that upwind schemes preserving steady states at rest enables it to very accurately compute near steady state flows. However, in [33] well-balanced WENO schemes for moving-water equilibria exhibited superior results over those preserving steady states at rest only. In this section, we test the ability of the scheme to converge to such solutions when the initial conditions are close to equilibrium. In order to simulate these conditions, we initialized the flow by confining water within the two ends of a channel connected to two reservoirs. The reservoirs hold water with identical value of $Q_{\text {in }}$ but different water heights $h_{\text {in }}$ and $h_{\text {out }}$. At time $t=0$, the water within the channel is let to flow and the solution is evolved over time to 

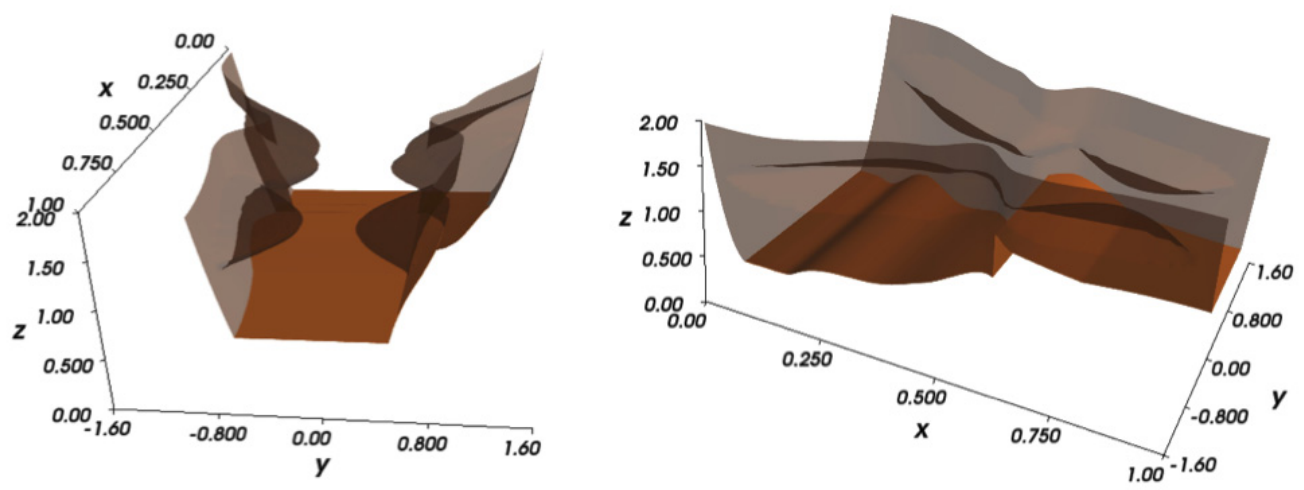

FIGURE 13. 3D-view of the channel's geometry.

observe whether it converges to the steady-state that is uniquely determined by the channels geometry and the data $Q_{\text {in }}$, and $h_{\text {out }}$; an exact solution that we compute before hand.

The correct implementation of the boundary conditions is essential to study the convergence of these flows to the unique steady flow determined by $Q_{\text {in }}$ and $h_{\text {out }}$. All the flows we consider are subcritical at the left inflow - boundary (i.e., $u-c<0$ ), thus for the numerical calculations, we specify the value of the discharge, $Q_{\text {left }}=Q_{\text {in }}$, at that boundary and the value of $h($ or $w)$ is extrapolated from the computational domain. At the right -outflow- boundary, if the flow is supercritical (i.e. $u-c>0$ ), both $h$ and $Q$ are extrapolated from the evolved solution inside the computational domain, otherwise, $Q$ is extrapolated and the total height is set to $w=w_{\text {out }}$.

\subsubsection{Subcritical flows}

The first steady flow that we investigate is a subcritical flow (i.e., its Froude number satisfies $F^{2}<1$ throughout the channel). The geometry of the channel is given by the width function (4.1) and a bottom topography consisting of a 3-bump spline with nodes $(x, z)=\{(0.2,0),(0.3,0.6),(0.4,0.4),(0.5,0.5)$, $(0.6,0.2),(0.7,0.3),(0.8,0)\}$. The flow invariants are set to $Q_{\text {in }}=2.0494$ and $w_{\text {out }}=1.5$. The flow is initially at rest and has total height $w_{\text {out }}$. Figure 16 shows the solution at time $t=20$. The dotted line on the top left of the figure is the numerical solution of the subcritical flow, which is in very good agreement with the exact solution (solid line). The solid black line on the left figure represents the topography. The top right of the figure shows the steady variables $Q$ and $E$, whose relative errors are $3.5 \times 10^{-3}$ and $1.2 \times 10^{-3}$ respectively. The 3D view of the subcritical flow is shown at the bottom of the figure. The topography is shown in light brown, walls in gray and the water surface in blue.

\subsubsection{Smooth transcritical flows}

In $[5,19]$ the effect of the channel's width and topography on steady-state flows was studied. Depending on the conditions at the outflow boundary, when a subcritical flow is accelerated through a contraction and/or a bump on the channel's floor, it may reach criticality (i.e., $F^{2}=1$ ) at some point within the channel. From that point on, the steady flow may remain smooth and supercritical or it may jump back to subcritical so as to match the outflow boundary conditions. In this test, we set $Q_{\text {in }}=0.4511$ and $w_{\text {out }}=0.1425$ for a channel whose width is given by $\sigma_{2}(x, z),(4.2)$, and bottom topography given by

$$
B(x)=\frac{1}{2}\left(1+\cos \left(\pi\left(x-\frac{1}{2}\right) / 0.4\right)\right) \chi_{[0.1,0.9]}(x) .
$$



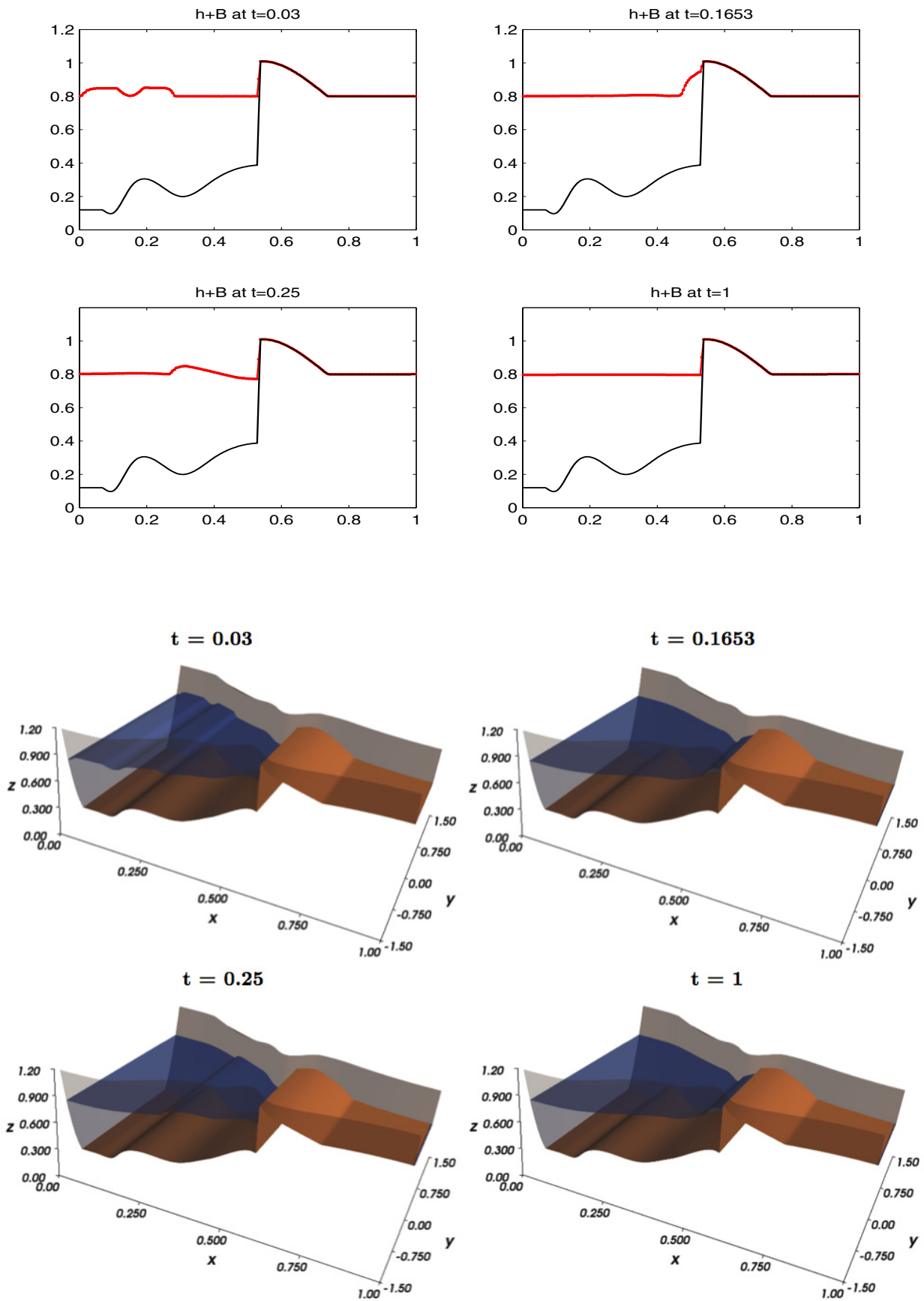

FigURE 14. Small perturbation to a wet-dry state at different times. Only a reflection is observed when the wave hits the discontinuity in the topography. 

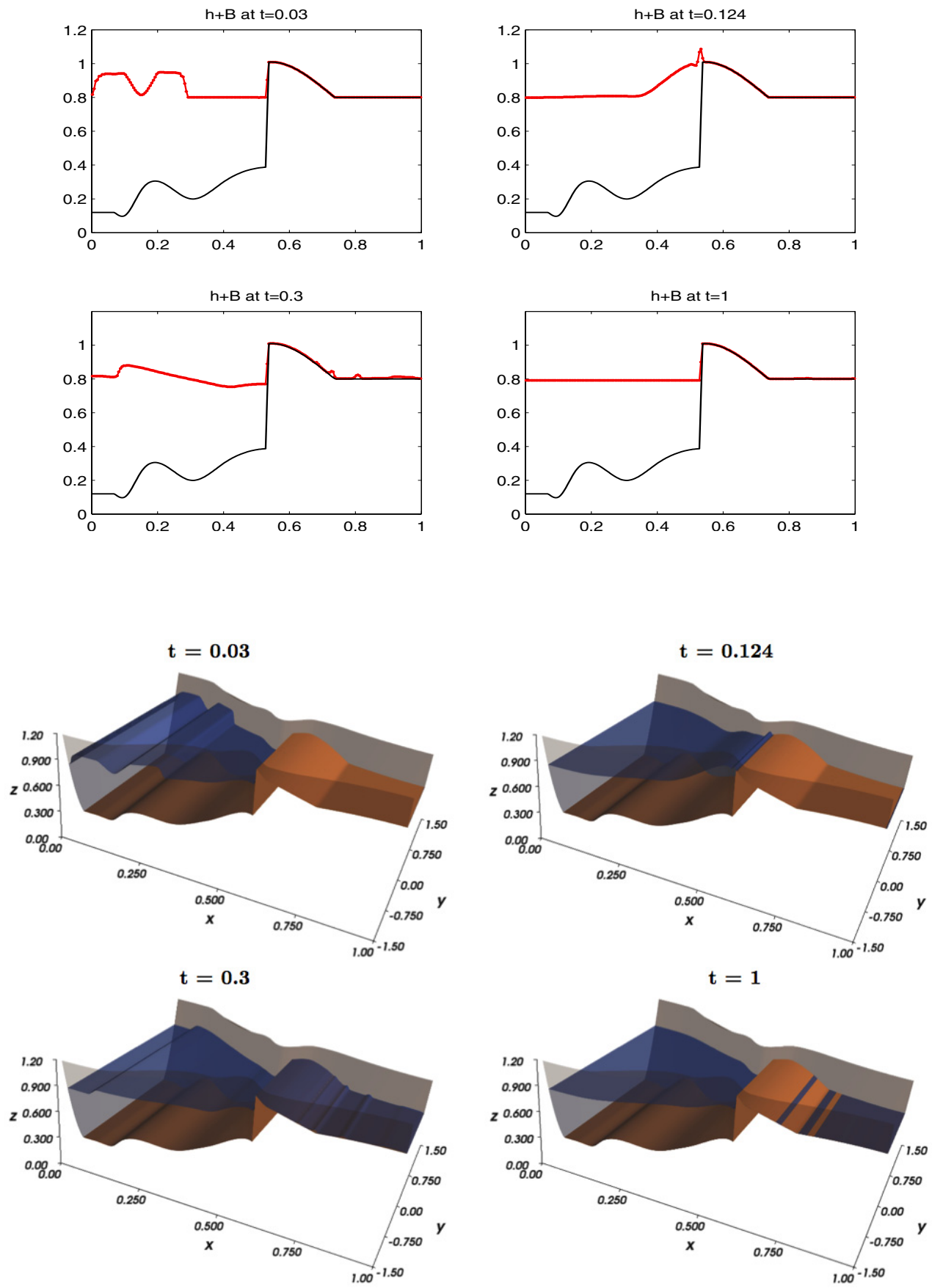

Figure 15. Large perturbation to a wet-dry state. The wave partially reflects back and partially transmits through the discontinuity when it hits it. 

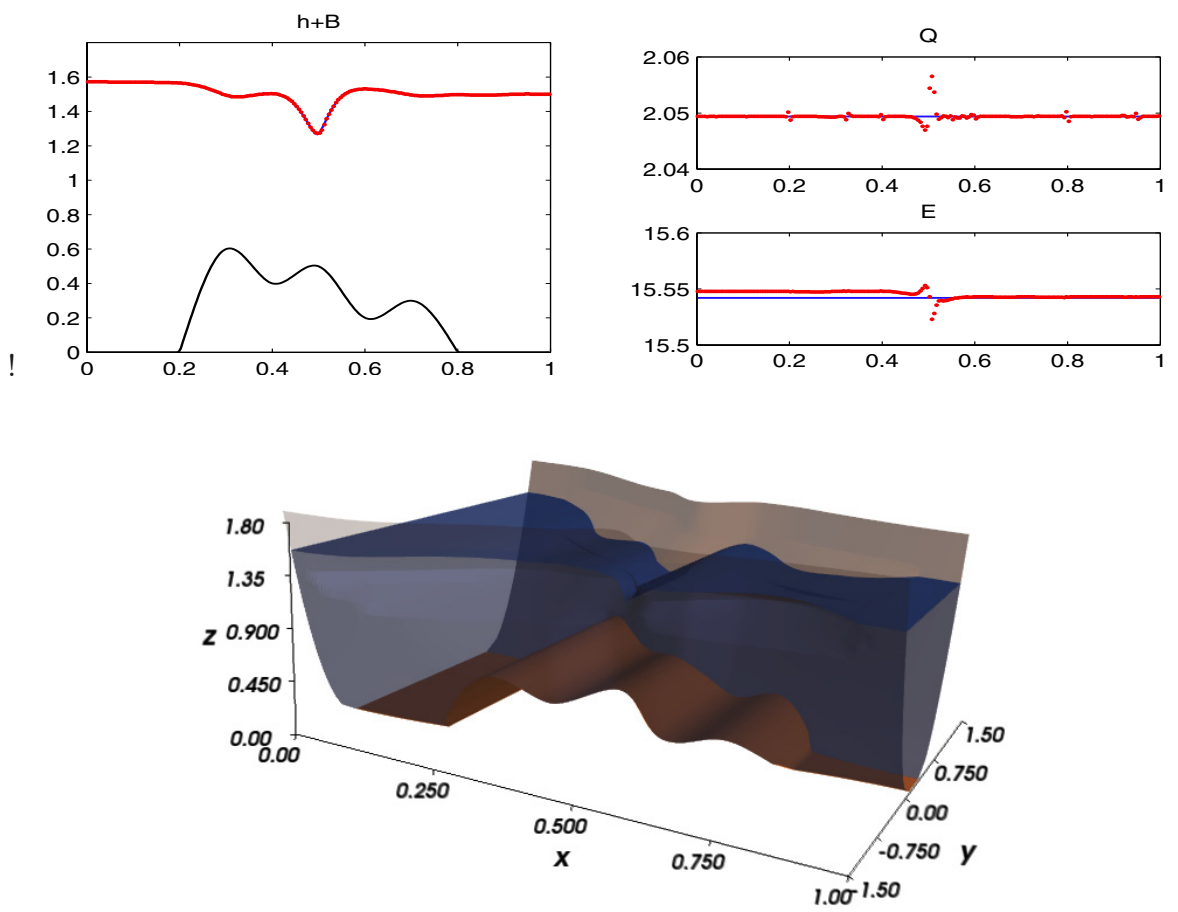

FiguRE 16. Steady state convergence at $t=20$ for subcritical flows. Top left shows the side view of the solutions, top right the steady variables $Q$ and $E$, and bottom shows a 3D view of the flow. The numerical approximation of the water level (dotted line) is in very good agreement.

Figure 17 shows the solution at time $t=2$. The dotted line on the top left of the figure is the numerical solution of the transcritical flow, which is in very good agreement with the exact solution (solid line). The solid black line represents the topography. The top right of the figure shows the computed values of the flow invariants $Q$ and $E$, whose relative errors with respect to the exact solution are $1.7 \times 10^{-3}$ and $6 \times 10^{-4}$ respectively. The $3 \mathrm{D}$ view of the transcritical flow is shown at the bottom of the figure; the topography is shown in light brown, the walls of the channel in gray, and the water surface in blue.

\subsubsection{Transcritical flow with shock}

Only certain boundary condition may be connected by smooth transcritical steady flows. If the conditions at the outflow boundary are those of a subcritical flow, then, the supercritical flow must dissipate energy through a stationary shock so as to match the outflow conditions. The jump occurs in the place where the jump conditions are satisfied, [1]. In this subsection we test the convergence to such flows over time. The boundary conditions consist of imposing $Q_{\text {in }}=0.4511$ at the inflow boundary, and $w_{\text {out }}=0.9769$ at the outflow. Figure 18 shows the solution at time $t=2$. The dotted line on the top left of the figure is the numerical solution of the transcritical flow, which is in very good agreement with the exact solution (solid line). The solid black line is the topography. The top right of the figure shows the steady variables $Q$ and $E$. The $3 \mathrm{D}$ view of the non smooth transcritical flow is shown at the bottom of the figure; the topography is shown in light brown, the walls of the channel in gray, and the water surface in blue.

\subsection{The oscillating lake}

In problems where the layer's depth is small, e.g., flooding problems or dam break simulations, numerical errors in the solution may lead to an unphysical negative depth of the water layer. With the following example we 

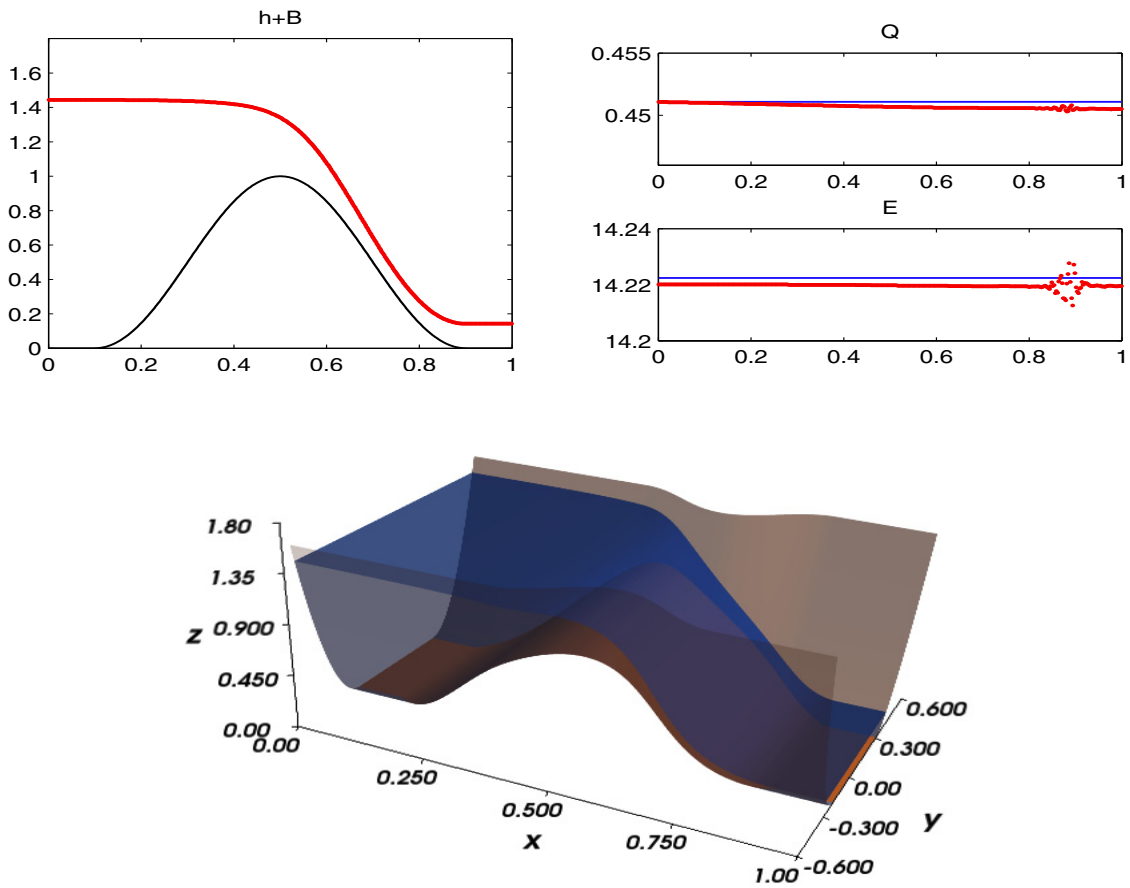

FiguRE 17. Steady state convergence at $t=2$ for smooth transcritical flows. Top left shows the flow profile, top right the steady variables $Q$ and $E$, and bottom shows the $3 \mathrm{D}$ view. The numerical approximation of the water level (dotted line) is in very good agreement.

test the positivity preserving property proved in proposition 3.3. On a channel whose geometry is given by (4.1) and a centered bump described by a spline of degree three with nodes $(-0.1,1.9),(0.3,1.2),(0.6,1.1),(0.7,1.5)$, $(0.8,1.2),(0.9,1.6),(1.1,1.8)$ (see Appendix B), we set initial conditions similar to those in the oscillating lake study presented in [5], and let the flow evolve over time.

Initially, the right side of the bump consists of only dry states. Figure 19 (top) shows the solution at times $t=0,0.15,0.6,20$. The top right snapshot shows how the flow starts to inundate the right side of the bump, which was initially dry. This forms two oscillating lakes interacting several times as the water jumps over the bump, spilling to the right side causing other oscillations until both sides reach a steady state at rest, as can be shown at $t=200$. The bottom of Figure 19 shows the 3D view of the corresponding flows.

\subsection{Dam break}

The last test simulates a dam break and is also aimed at testing the positivity preserving property of our central scheme. The topography is the 3-bump spline given in Section 4.4.1 and the initial conditions consist of a steady-state of rest with total height $w=0.8$. We apply reflecting boundary conditions at the (left) inflow boundary and impose $w_{\text {out }}=10^{-3}$ at the (right) outflow boundary. Figure 20 shows the evolution of the drainage simulation. We observe that the water drains through the right boundary, except for the areas where the water gets trapped between the bumps.

\section{Conclusions:}

A model for shallow water flows in channels with arbitrary cross-sectional area was presented and its properties discussed. Taking these properties into consideration, we designed a positivity preserving high-resolution, 

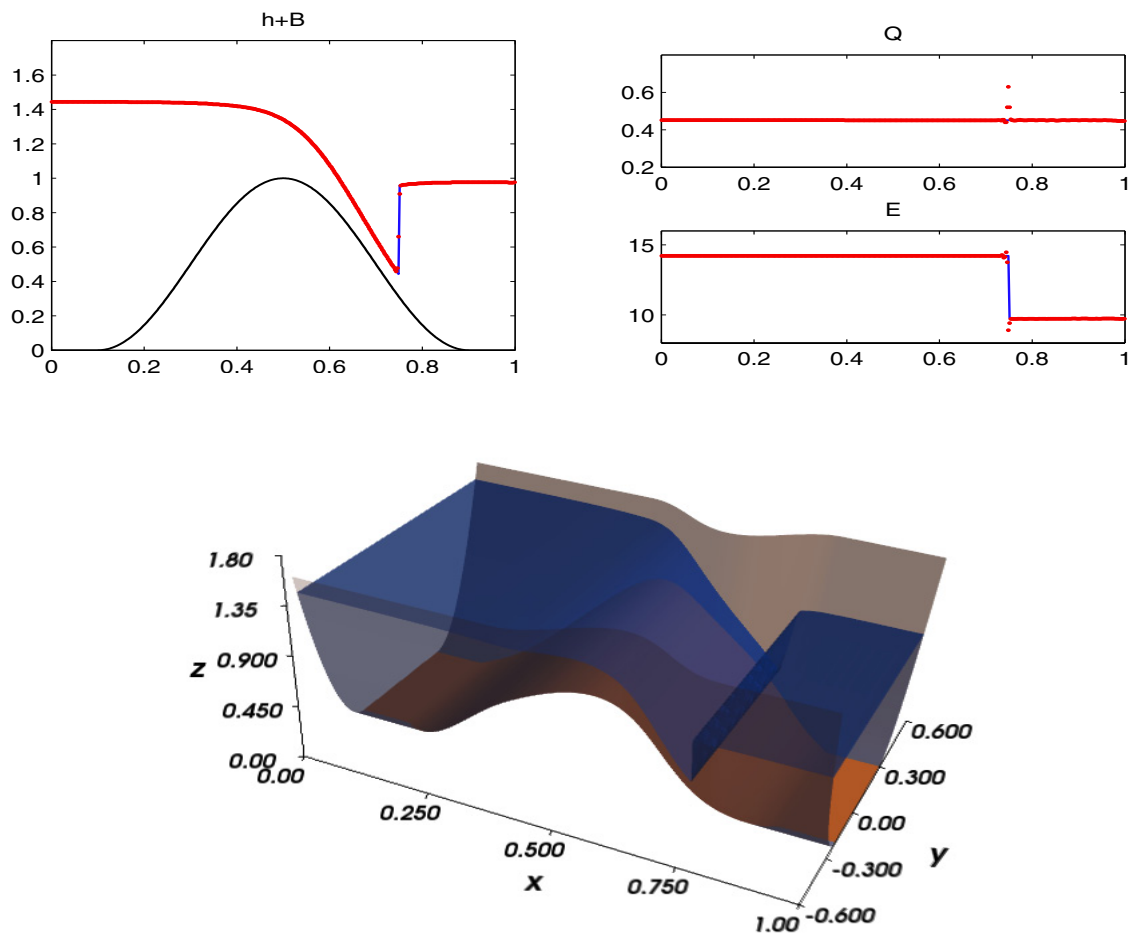

FIGURE 18. Steady-state convergence at $t=2$ for transcritical flows with shock. Top left shows the side view of the solutions, top right the steady variables $Q$ and $E$, and bottom shows the 3D view. The numerical approximation of the water level (dotted line) is in very good agreement.

non-oscillatory semi-discrete central scheme for simulating the flows described by the model, and we proved that the scheme enjoys several desirable properties for computing these solutions: the well balance property was achieved by finding a consistent discretization of the source term that balances the flux gradient when the flow is at rest; a reconstruction from the cell averages of the data that preserves the positivity of the layer's depth at the cell interfaces together with a $C F L$ restriction guarantees the positivity preserving property; and the ability to detect and resolve partially wet regions was built into the scheme by introducing a modification in the numerical flux and the estimate of the speed of propagation. Several numerical experiments were presented so as to demonstrate the robustness of the numerical algorithm, and its ability to capture steady-flows and resolve wet-dry fronts.

\section{Appendix A. Deconvolution of Water elevation from total Area}

In order to recover the cell average of the total water elevation, $\bar{w}_{j}$, from the updated total area, $\bar{A}_{j}^{T}$ after each time step, we make use of the linearization of the channel illustrated in Figure 1. That is, if $\sigma(x, z)$ is a piecewise linear function in the vertical direction with nodes $\left(z_{k}, \beta_{k}(x)\right), 0=z_{0}<z_{1}<z_{2}<\ldots<z_{k}<z_{k+1}<\ldots$, we define

$$
\sigma(x, z)=\beta_{k}(x)+m_{k}(x)\left(z-z_{k}\right), \quad \text { for } z_{k} \leq z<z_{k+1},
$$

with the slope, $m_{k}(x)$, given by

$$
m_{k}(x)=\frac{\beta_{k+1}(x)-\beta_{k}(x)}{z_{k+1}-z_{k}} .
$$



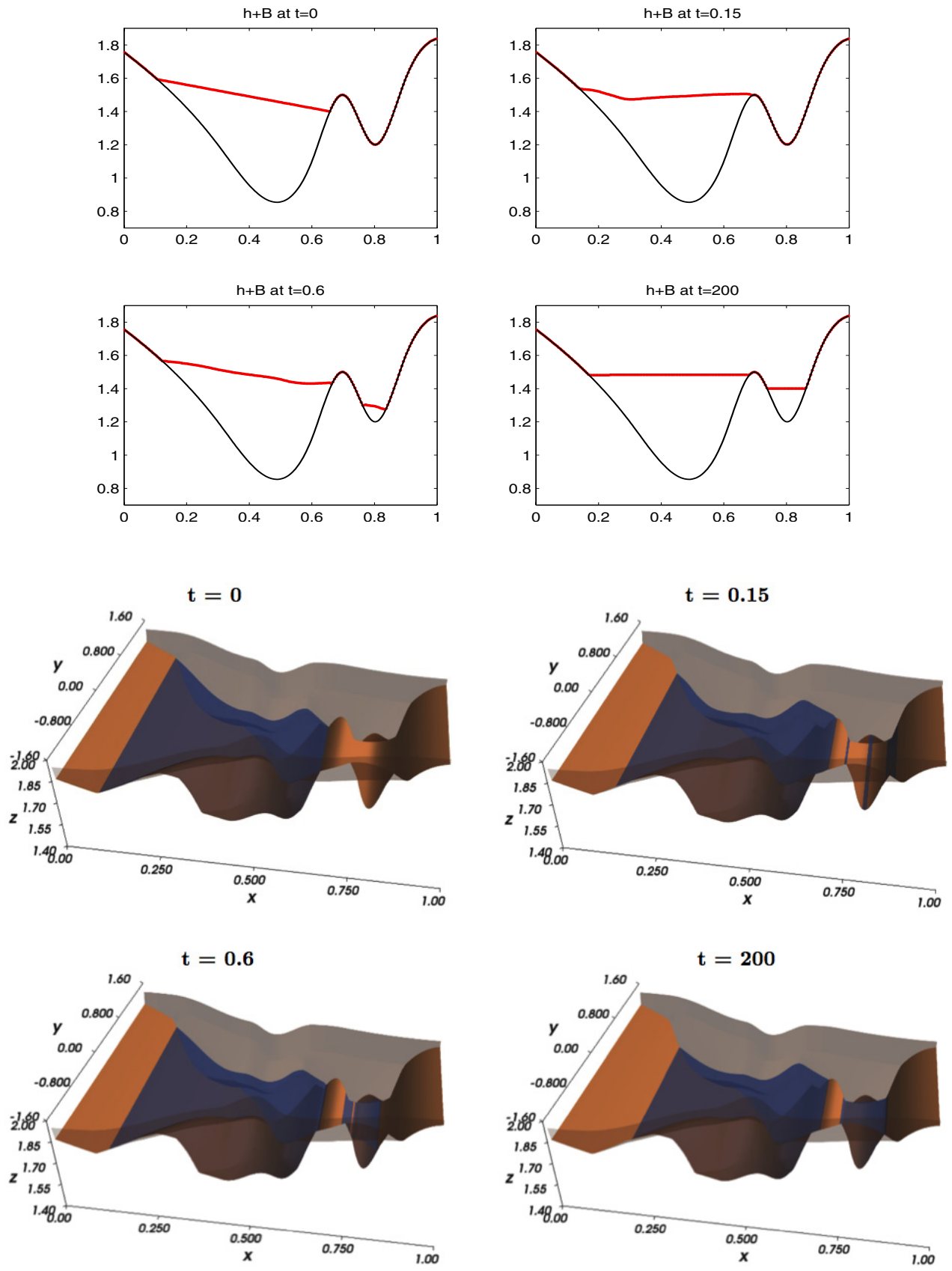

Figure 19. Numerical solution for an oscillating lake at times $t=0,0.15,0.6,20$. 

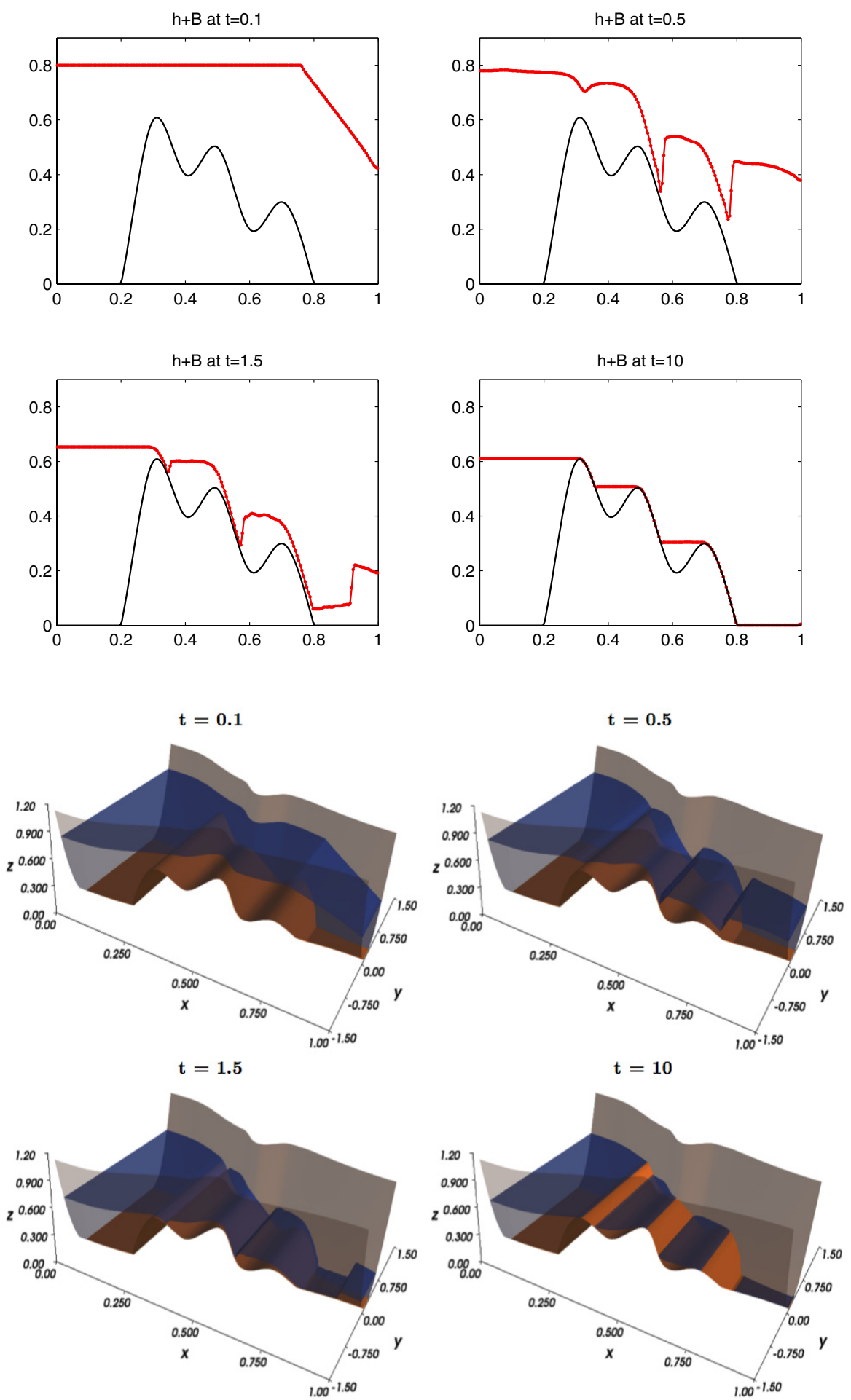

Figure 20. Dam break simulation at times $t=0.1,0.5,1.5,10$. 
Then the deconvolution consists of solving the quadratic polynomial in $\bar{w}_{j}$

$$
\left(\beta_{k}\left(x_{j}\right)+m_{k}\left(x_{j}\right) \frac{\bar{w}_{j}-z_{k}}{2}\right)\left(\bar{w}_{j}-z_{k}\right)=\bar{A}_{j}^{T}-\int_{B\left(x_{j}\right)}^{z_{k}} \sigma\left(x_{j}, z\right) \mathrm{d} z
$$

where $k$ is such that $z_{k} \leq \bar{w}_{j}<z_{k+1}$.

\section{Appendix B. Topographies AND CUBIC SPline Interpolations}

The topographies used in different examples in the paper are obtained using standard cubic spline interpolations. For the convenience of the reader, the algorithm is described here. Splines of degree three interpolate $n+1$ nodes $\left(x_{0}, z_{0}\right),\left(x_{1}, z_{1}\right), \ldots\left(x_{n}, z_{n}\right)$ with the aid of cubic polynomials. More precisely, and assuming without loss of generality that $x_{0}<x_{1}<\ldots x_{n}$, the topography is defined as

$$
B(x)=p(x):= \begin{cases}p_{0}(x), & x_{0} \leq x \leq x_{1} \\ p_{1}(x), & x_{1} \leq x \leq x_{2} \\ \vdots & \\ p_{n-1}(x), & x_{n-1} \leq x \leq x_{n},\end{cases}
$$

where each $p_{i}, i=0, \ldots, n$ is a cubic polynomial satisfying the following conditions

$$
\begin{array}{ll}
p_{i}\left(x_{i}\right)=z_{i}, & 0 \leq i \leq n-1, \text { and } p_{n-1}\left(x_{n}\right)=y_{n}, \\
p_{i-1}\left(x_{i}\right)=p_{i}\left(x_{i}\right), & 1 \leq i \leq n-1 \\
p_{i-1}^{\prime}\left(x_{i}\right)=p_{i}^{\prime}\left(x_{i}\right), & 1 \leq i \leq n-1 \\
p_{i-1}^{\prime \prime}\left(x_{i}\right)=p_{i}^{\prime \prime}\left(x_{i}\right), & 1 \leq i \leq n-1 \\
p^{\prime \prime}\left(x_{0}\right)=p^{\prime \prime}\left(x_{n}\right)=0, &
\end{array}
$$

where the primes denote derivatives with respect to $\mathrm{x}$. This gives $4 n$ conditions and $4 n$ unknowns for the coefficients of the cubic polynomials.

To solve the system, let us define $r_{i}=S^{\prime \prime}\left(x_{i}\right)$. Since we impose the condition $S^{\prime \prime}\left(x_{0}\right)=S^{\prime \prime}\left(x_{n}\right)=0$, then $r_{0}=r_{n}=0$. Let $h_{i}=x_{i+1}-x_{i}$, and $d_{i}=\frac{z_{i+1}-z_{i}}{h_{i}}$. The cubic polynomials can be written as

$$
p_{i}(x)=\frac{r_{i+1}}{6 h_{i}}\left(x-x_{i}\right)^{3}+\frac{r_{i}}{6 h_{i}}\left(x_{i+1}-x\right)^{3}+\left(\frac{z_{i+1}}{h_{i}}-\frac{h_{i}}{6} r_{i+1}\right)\left(x-x_{i}\right)+\left(\frac{z_{i}}{h_{i}}-\frac{h_{i}}{6} r_{i}\right)\left(x_{i+1}-x\right),
$$

where the unknowns $r_{i}, 1 \leq i \leq n$ can be computed by solving the system

$$
\left(\begin{array}{cccccc}
\frac{h_{0}+h_{1}}{3} & \frac{h_{1}}{6} & & & & \\
\frac{h_{1}}{6} & \frac{h+1+h_{2}}{3} & \frac{h_{2}}{6} & & & \\
& \frac{h_{2}}{6} & \frac{h_{2}+h_{3}}{3} & \frac{h_{3}}{6} & & \\
& & \ddots & \ddots & \ddots & \\
& & & \frac{h_{n-3}}{6} & \frac{h_{n-3}+h_{n-2}}{3} & \\
& & & h_{n-2} \\
& & \frac{h_{n-2}}{6} & \frac{h_{n-2}+h_{n-1}}{3}
\end{array}\right)\left(\begin{array}{c}
r_{1} \\
r_{2} \\
r_{3} \\
\vdots \\
r_{n-2} \\
r_{n-1}
\end{array}\right)=\left(\begin{array}{c}
d_{1}-d_{0} \\
d_{2}-d_{1} \\
d_{3}-d_{2} \\
\vdots \\
d_{n-2}-d_{n-3} \\
d_{n-1}-d_{n-2}
\end{array}\right)
$$

The system above involves a tridiagonal matrix. The Thomas method can be used to solve it.

Acknowledgements. Part of this work was done while Gerardo Hernandez-Duenas was still at the University of Michigan as a Ph.D. student, and both authors would like to thank Prof. Smadar Karni for helpful discussions. Part of this work was done during the participation of both authors at the program Model and Data Hierarchies for Simulating and Understanding Climate hosted by IPAM (UCLA) during the spring of 2010. The hospitality and generosity of IPAM and the organizers of the program is gratefully acknowledged. 


\section{REFERENCES}

[1] R. Abgrall and S. Karni, Two-layer shallow water system: a relaxation approach. SIAM J. Sci. Comput. 31 (2009) $1603-1627$.

[2] L. Armi, The hydraulics of two flowing layers with different densities. J. Fluid Mech. 163 (1986) 27-58.

[3] L. Armi and D.M. Farmer, Maximal two-layer exchange through a contraction with barotropic net flow. J. Fluid Mech. 186 (1986) $27-51$.

[4] E. Audusse, F. Bouchut, M.-O. Bristeau, R. Klein and B. Perthame, A fast and stable well-balanced scheme with hydrostatic reconstruction for shallow water flows. SIAM J. Sci. Comput. 25 (2004) 2050-2065.

[5] J. Balbás and S. Karni, A central scheme for shallow water flows along channels with irregular geometry. ESAIM: M2AN 43 (2009) 333-351.

[6] A. Bollermann, G. Chen, A. Kurganov and S. Noelle, A well-balanced reconstruction of wet/dry fronts for the shallow water equations. J. Sci. Comput. (2011) 1-24.

[7] A. Bollermann, S. Noelle and M. Lukáčová-Medvid'ová, Finite volume evolution Galerkin methods for the shallow water equations with dry beds. Commun. Comput. Phys. 10 (2011) 371-404.

[8] F. Bouchut, Nonlinear stability of finite volume methods for hyperbolic conservation laws and well-balanced schemes for sources. Frontiers in Mathematics. Birkhäuser Verlag, Basel (2004).

[9] M. Castro, J. Macías and C. Parés, A $Q$-scheme for a class of systems of coupled conservation laws with source term. Application to a two-layer 1-D shallow water system. ESAIM: M2AN 35 (2001) 107-127.

[10] M.J. Castro, J.A. García-Rodríguez, J.M. González-Vida, J. Macías, C. Parés and M.E. Vázquez-Cendón, Numerical simulation of two-layer shallow water flows through channels with irregular geometry. J. Comput. Phys. 195 (2004) $202-235$.

[11] M.J. Castro, A. Pardo Milanés and C. Parés, Well-balanced numerical schemes based on a generalized hydrostatic reconstruction technique. Math. Models Methods Appl. Sci. 17 (2007) 2055-2113.

[12] N. Črnjarić-Žic, S. Vuković and L. Sopta, Balanced finite volume WENO and central WENO schemes for the shallow water and the open-channel flow equations. J. Comput. Phys. 200 (2004) 512-548.

[13] D.M. Farmer and L. Armi, Maximal two-layer exchange over a sill and through the combination of a sill and contraction with barotropic flow. J. Fluid Mech. 164 (1986) 53-76.

[14] P. Garcia-Navarro and M.E. Vazquez-Cendon, On numerical treatment of the source terms in the shallow water equations. Comput. Fluids 29 (2000) 951-979.

[15] D.L. George, Augmented Riemann solvers for the shallow water equations over variable topography with steady states and inundation. J. Comput. Phys. 227 (2008) 3089-3113.

[16] S. Gottlieb, C.-W. Shu and E. Tadmor, Strong stability-preserving high-order time discretization methods. SIAM Review 43 (2001) 89-112.

[17] G. Hernández-Dueñas and S. Karni, Shallow water flows in channels. J. Sci. Comput. 48 (2011) 190-208.

[18] S. Jin, A steady-state capturing method for hyperbolic systems with geometrical source terms. ESAIM: M2AN (2001) 35 631-645.

[19] S. Karni and G. Hernández-Dueñas, A scheme for the shallow water flow with area variation. AIP Conference Proceedings. Vol. 1168 of International Conference Numer. Anal. Appl. Math., Rethymno, Crete, Greece. American Institute of Physics (2009) 1433-1436.

[20] A. Kurganov and D. Levy, Central-upwind schemes for the Saint-Venant system. ESAIM: M2AN 36 (2002) $397-425$.

[21] A. Kurganov and G. Petrova, A second-order well-balanced positivity preserving central-upwind scheme for the Saint-Venant system. Commun. Math. Sci. 5 (2007) 133-160.

[22] A. Kurganov and G. Petrova, Central-upwind schemes for two-layer shallow water equations. SIAM J. Sci. Comput. 31 (2009) $1742-1773$.

[23] A. Kurganov and E. Tadmor, New high-resolution central schemes for nonlinear conservation laws and convection-diffusion equations. J. Comput. Phys. 160 (2000) 241-282.

[24] R.J. LeVeque, Balancing source terms and flux gradients in high-resolution Godunov methods: the quasi-steady wavepropagation algorithm. J. Comput. Phys. 146 (1998) 346-365.

[25] S. Noelle, N. Pankratz, G. Puppo and J.R. Natvig, Well-balanced finite volume schemes of arbitrary order of accuracy for shallow water flows. J. Comput. Phys. 213 (2006) 474-499.

[26] S. Noelle, Y. Xing and C.-W. Shu, High-order well-balanced finite volume WENO schemes for shallow water equation with moving water. J. Comput. Phys. 226 (2007) 29-58.

[27] B. Perthame and C. Simeoni, A kinetic scheme for the Saint-Venant system with a source term. Calcolo 38 (2001) $201-231$.

[28] P.L. Roe, Upwind differencing schemes for hyperbolic conservation laws with source terms. Nonlinear hyperbolic problems (St. Etienne, 1986). In vol. 1270 of Lecture Notes in Math. Springer, Berlin (1987) 41-51.

[29] G. Russo, Central schemes for balance laws. Hyperbolic problems: theory, numerics, applications, Vol. I, II (Magdeburg, 2000). In vol. 140 of Internat. Ser. Numer. Math. Birkhäuser, Basel (2001) 821-829.

[30] B. van Leer, Towards the ultimate conservative difference scheme. V. A second-order sequel to Godunov's method. J. Comput. Phys. 32 (1979) 101-136; J. Comput. Phys. 135 (1997) 227-248.

[31] M. E. Vázquez-Cendón. Improved treatment of source terms in upwind schemes for the shallow water equations in channels with irregular geometry. J. Comput. Phys. 148 (1999) 497-526. 
[32] S. Vuković and L. Sopta, High-order ENO and WENO schemes with flux gradient and source term balancing. In Applied mathematics and scientific computing (Dubrovnik, 2001). Kluwer/Plenum, New York (2003) 333-346.

[33] Yulong Xing, Chi-Wang Shu and Sebastian Noelle, On the advantage of well-balanced schemes for moving-water equilibria of the shallow water equations. J. Sci. Comput. 48 (2011) 339-349. 